\title{
Gene Expression Profiling Shows That NbFDN1 Is Involved in Modulating the Hypersensitive Response-Like Cell Death Induced by the Oat dwarf virus RepA Protein
}

\author{
Huwei Hou, ${ }^{1}$ Ya Hu, ${ }^{1}$ Qian Wang, ${ }^{1}$ Xiongbiao Xu, ${ }^{1}$ Yajuan Qian, ${ }^{1,+}$ and Xueping Zhou, ${ }^{1,2}$ \\ ${ }^{1}$ State Key Laboratory of Rice Biology, Institute of Biotechnology, Zhejiang University, Hangzhou 310058, People's Republic of \\ China; and ${ }^{2}$ State Key Laboratory for Biology of Plant Diseases and Insect Pests, Institute of Plant Protection, Chinese Academy \\ of Agricultural Sciences, Beijing, 100193, People's Republic of China
}

Accepted 8 April 2018.

In this study, we used high-throughput deep nucleotide sequencing to characterize the global transcriptional response of Nicotiana benthamiana plants to transient expression of the RepA protein from Oat dwarf virus (ODV). We identified 7,878 significantly differentially expressed genes (DEG) that mapped to 125 pathways, suggesting that comprehensive networks are involved in regulation of RepA-induced cell death. Of the 202 DEG associated with photosynthesis, expression of 195 was found to be downregulated, indicating a significant inhibition of photosynthesis in response to RepA expression, which is associated with chloroplast disruption and physiological changes. We focused our analysis on NbFDN1, a member of the ferredoxin protein family that participates in the chloroplast electron transport chain performing oxygenic photosynthesis, which was identified to directly interact with NbTsip1. We separately knocked down the expression of NbFDN1 and NbTsip1 using virus-induced gene silencing, and found that NbFDN1 silencing speeded up the development of RepAinduced cell death, unlike $N b T$ sipl 1 silencing, which showed an opposite effect on RepA-induced response. Further study showed increased $\mathrm{H}_{2} \mathrm{O}_{2}$ accumulation and a negative correlation between the transcripts of $N b F D N 1$ and NbTsip1 in NbFDN1-silenced plants. Hence, we speculate that NbFDN1 has an effect on RepA-induced hypersensitive response-like response by modulating $\mathrm{NbT}$ sip1 transcription as well as $\mathrm{H}_{2} \mathrm{O}_{2}$ production.

Plants have to endure many diverse abiotic and biotic stresses and, therefore, have evolved elaborate mechanisms to monitor and respond to environmental changes. One example is an incompatible immune reaction known as the hypersensitive response (HR), by which plants form a physical barrier to prevent further pathogen infection (Pontier et al. 1994). Usually, HRlike responses are produced by viral infection (Chen et al. 2017; Kim et al. 2008). In some cases, the effects of viral proteins on physiological conditions within the infected host tissues can

${ }^{\dagger}$ Corresponding author: Y. Qian; E-mail: yjqian@zju.edu.cn

Funding: This work was supported by the National Natural Science Foundation of China (grant numbers 31671997 and 31390421).

*The $\boldsymbol{e}$-Xtra logo stands for "electronic extra" and indicates that five supplementary figures and five supplementary tables are published online.

@ 2018 The American Phytopathological Society induce HR-mediated cell death (Angel and Schoelz 2013; Hu et al. 2012; Kim et al. 2010). Upon the introduction of various HR elicitors, plant metabolism must balance potentially competing demands for resources to support defense against pathogen infection. Hence, a massive reprogramming of plant gene expression and hormonal and chemical defense responses is initiated (Brading et al. 2000; Oka et al. 2013; Pandey et al. 2010). A decline in photosynthetic rate following pathogen attack is well documented (Bilgin et al. 2010). In addition, examinations of the plant transcriptome have revealed that many photosynthetic genes are downregulated following biotrophic pathogens attack (Bozsó et al. 2009; Geng et al. 2017; Sood and Chauhan 2017).

Oat dwarf virus (ODV) is a member of the genus Mastrevirus in the family Geminiviridae and is transmitted by leafhoppers (Schubert et al. 2007). Mastreviruses have a monopartite genome and mainly infect monocotyledonous plant species. Four proteins are encoded by the viral genome of ODV: the movement protein (from the V2 open reading frame [ORF]) and the capsid protein (from the V1 ORF) on the viral sense strand, and two replication-initiator proteins, Rep (spliced from the $\mathrm{C} 1$ and C2 ORF) and RepA (C1 ORF alone) on the complementary strand. The Rep and RepA proteins share the same $\mathrm{N}$ terminus but differ in their $\mathrm{C}$ termini, which allows for their different and multiple functions in the viral life cycle (Collin et al. 1996; Luque et al. 2002; Xie et al. 1995). Recently, we demonstrated that the RepA protein encoded by ODV acts as an elicitor of HR-like cell death (Qian et al. 2016). However, the molecular mechanism underlying the complex interaction between ODV RepA and the host plant defense system to elicit an HR-like response is poorly understood.

High-throughput gene expression analysis using massive RNA sequencing (RNA-Seq) is far more precise and sensitive for the characterization of transcriptomes when compared with other methods of measuring mRNA levels (Wang et al. 2009), and has been used as a valuable tool to study plant-pathogen interactions (Kawahara et al. 2012; Martin et al. 2016; Nejat et al. 2015; Qin et al. 2017; Rodamilans et al. 2014; Rodrigues et al. 2013). In this study, we utilized high-throughput deep sequencing to characterize the global transcriptional response of Nicotiana benthamiana plants to transient expression of RepA and RepA ${ }^{\mathrm{UT}}$ (an untranslatable mutant, included as a control) derived from ODV. Compared with the control, the differentially expressed genes (DEG) from several pathways, including photosynthesis, have been characterized, and suggest a group of candidate genes related to RepA-induced cell death. Using Tobacco rattle virus (TRV)-based virus-induced gene 
silencing (VIGS), we demonstrate that silencing of $N b F D N 1$, a member of the ferredoxin $(\mathrm{Fd})$ protein family that functions as an electron acceptor on the stromal side of the chloroplast electron transport chain performing oxygenic photosynthesis (Hanke and Mulo 2013), accelerated HR-type cell death induced by RepA. This research will enhance our understanding of the mechanisms of the HR-like response in model plants such as $N$. benthamiana.

\section{RESULTS}

\section{RNA-Seq analysis of the gene expression profile influenced by ODV RepA.}

Previously, we reported that the transient expression of ODV RepA could induce an HR-like cell death response in tobacco plants. In order to better understand the molecular mechanisms underlying RepA-induced cell death, comparative analysis of changes in gene transcription was performed to explore the effects of RepA on host global gene expression. N. benthamiana plants were agroinoculated with two constructs, 35S-RepA and $35 \mathrm{~S}-\operatorname{RepA}^{\mathrm{UT}}$ (an untranslatable mutant control). Samples (called S-RepA and S-RepA ${ }^{\mathrm{UT}}$ ) were collected at $72 \mathrm{~h}$ postinoculation (hpi) and subjected to Illumina RNA sequencing. After removing the low-quality reads and adaptor sequences, 11,667,483 and $12,280,703$ clean reads, respectively, remained from the two different samples (Supplementary Table S1). Of the total reads, the proportion of mapped reads ranged from $85.42 \%\left(\mathrm{~S}-\mathrm{RepA}^{\mathrm{UT}}\right)$ to $86.43 \%$ (S-RepA).

The transcriptome reference database for $N$. benthamiana was used to map the clean RNA-Seq reads from S-RepA and S$\operatorname{RepA}^{\mathrm{UT}}$. The percentage of genes identified increased with the number of reads to $56.17 \%$ (S-RepA ${ }^{\mathrm{UT}}$ ) and $59.77 \%$ (S-RepA), and eventually reached a plateau, implying full saturation of the transcriptome (Supplementary Fig. S1). In total, 41,367 unigenes (S-RepA) and 43,257 unigenes (S-RepA ${ }^{\mathrm{UT}}$ ) were identified. The "gene coverage" of these unigenes ranged from 0.60 to $99.94 \%$, showing that there was no bias between S-RepA and S-RepA ${ }^{\text {UT }}$ (Supplementary Fig. S2). In addition, the levels of gene expression for most of the unique genes normalized by the reads per kilobase of transcript per million mapped reads (RPKM) method were from -0.5 to 1.5 ( $\log _{10}$ ratio) for S-RepA and S-RepA ${ }^{\mathrm{UT}}$ (Supplementary Fig. S3).

\section{DEG in response to RepA expression.}

To identify genes that showed significant changes in expression in response to the RepA elicitor, we analyzed the DEG between the S-RepA and S-RepA ${ }^{\mathrm{UT}}$ samples. An absolute value $\left(\log _{2}\right.$ ratio $\left.\geq 1\right)$ with a false discovery rate $(\mathrm{FDR})<$ 0.001 was used as the threshold to identify the DEG. There were 13,384 genes in which transcription levels showed significant difference in the S-RepA versus S-RepA ${ }^{\mathrm{UT}}$ pairwise analysis; 4,898 genes were upregulated and 8,486 were downregulated (Fig. 1A), revealing the association of a comprehensive group of DEG with the RepA expression. Interestingly, of the DEG, the number of upregulated genes $(36.6 \%)$ was much less than the number of downregulated genes $(63.4 \%)$. The detected fold changes ( $\log _{2}$ ratio) of relative gene expression ranged from -14.3 to 13.9 , and more than $84 \%$ of the genes were up- or downregulated between 1.0- and 5.0-fold, accounting for 7,182 downregulated genes (53.6\%) and 4,101 upregulated genes (30.6\%) (Fig. 1B). Expression of only approximately 9.2\% $(1,226)$ of the genes was up- or downregulated between 5.0and 10.0-fold (Fig. 1B). Results of correlation analysis showed that Pearson's correlation coefficient for S-RepA versus S-RepA ${ }^{\mathrm{UT}}$ was low $(r=0.34)$ (Fig. 1C), suggesting the large effect of RepA transient expression on the gene expression profile of $N$. benthamiana.
To validate the DEG data, we compared the gene expression profiles of the S-RepA and S-RepA ${ }^{\mathrm{UT}}$ samples using quantitative reverse-transcription polymerase chain reaction (qRT-PCR) assays. Of 41 genes selected randomly, 39 genes showed a concordant direction of change in expression for both the differential gene expression (using RNA-Seq data) and qRTPCR analyses (Fig. 1D; Supplementary Table S2). Our results are consistent with previous reports that describe the discrepancies in the data obtained from RNA-Seq and qRT-PCR. The differences observed may be caused by the lower sensitivity of qRT-PCR compared with RNA-Seq. Nevertheless, our qRT-PCR analysis confirmed the direction of change in expression detected by RNA-Seq analysis, indicating that the DEG data are generally reliable.

\section{Functional analysis of the DEG.}

To further understand the host responses that are induced by RepA expression in $N$. benthamiana, gene ontology (GO) was used to classify the 13,384 DEG in the S-RepA versus $\mathrm{S}-\mathrm{RepA}^{\mathrm{UT}}$ pairwise analysis. In total, 49 significantly different GO terms were clustered in the three main GO functional categories (biological process, cellular component, and molecular function) (Supplementary Fig. S4). Within the biological process class, a large number of DEG were enriched in the "metabolic process", "cellular process", and "response to stimulus" GO terms. In addition, GO terms found to specifically participate in cells, cell parts, organelles, and membranes were dominant within the cellular component class. In contrast, a high proportion of DEG from the GO terms "binding activity", "catalytic activity", and "transporter activity" belonged to the molecular function class.

In addition to GO term assignments, all of the DEG were mapped using the Kyoto Encyclopedia of Genes and Genomes (KEGG) database. There were 7,878 significantly changed DEG with KEGG annotation that mapped to 125 pathways (Supplementary Table S3). Among the KEGG pathways, metabolic pathways and biosynthesis of secondary metabolites were the main functional annotated groups (Table 1). It is noteworthy that the defense-related pathways with the most significant DEG enrichment for S-RepA versus S-RepA ${ }^{\text {UT }}$ were "plant hormone signal transduction", "plant-pathogen interaction", and "phenylpropanoid biosynthesis" (Table 1). In the KEGG pathway "plant hormone signal transduction", 234 genes were upregulated and 346 were downregulated. In total, 282 genes were upregulated and 274 were downregulated in the KEGG pathway "plant-pathogen interaction". In the KEGG pathway "phenylpropanoid biosynthesis", the expression of 213 genes was altered significantly, and included 117 upregulated genes and 96 downregulated genes. Ubiquitination is a key posttranslational protein modification that plays an important role in the plant immune system against invasion by pathogens. In the S-RepA versus S-RepA ${ }^{\mathrm{UT}}$ comparison, KEGG pathway enrichment analysis showed that the pathway "ubiquitin mediated proteolysis" was significantly enriched in the DEG. Of the 115 DEG belonging to this pathway, 69 genes were upregulated and 46 were downregulated.

\section{Expression of photosynthesis-related pathway genes is strongly suppressed by $\operatorname{RepA}$ expression.}

The photosynthetic fixation of carbon dioxide is divided into the light and dark reactions. Several reports have shown that photosynthesis is a primary target of different types of biotic stress (Rahoutei et al. 2000; Ryšlavá et al. 2003). It is worth mentioning that the DEG in the S-RepA versus S-RepA ${ }^{\text {UT }}$ comparison include a large number of genes associated with photosynthesis, suggesting a striking interaction between photosynthesis and the RepA-induced HR-like response. Of 
the 140 DEG associated with light reactions, 136 genes were downregulated compared with the control (Table 2), indicating significant inhibition of photosynthesis in response to RepA expression. It is intriguing that the expression of genes related to the electron transport chain and ATP synthesis were all downregulated. In addition, the genes encoding the major components of the photosystem I (PSI) and photosystem II (PSII) reaction centers were also markedly reduced in expression in the $N$. benthamiana leaves agroinoculated with 35S-RepA. Further analysis of DEG associated with dark reactions showed that, of the $62 \mathrm{DEG}$ associated with the Calvin-Benson cycle, 59 genes were downregulated compared with the control (Fig. 2A; Supplementary Table S4).

The linkage between photosynthesis and transient RepA expression was further demonstrated by observation of chlorophyll fluorescence parameters. The data showed that the maximum photochemical efficiency of PSII (Fv/Fm), the actual photochemical efficiency of PSII (Y(II)), and the electron transfer rate (ETR) exhibited marked decreases after RepA expression in leaf tissues at 48 hpi compared with those of the $35 \mathrm{~S}-\mathrm{RepA}^{\mathrm{UT}}$-inoculated sample and the wild-type control (Fig. 2B to E). Obviously, RepA expression impairs photosynthesis in the plant. However, interestingly, there were no


Fig. 1. Analysis of differentially expressed genes (DEG) derived from the S-RepA versus S-RepA ${ }^{\mathrm{UT}}$ comparison. A, Summary of the numbers of DEG. False discovery rate $<0.001$ and the absolute value of $\log _{2}$ ratio $\geq 1$ were used as the threshold to judge the significance of differences in gene expression. $R P K M=$ reads per kilobase of transcript per million mapped reads. B, Relative fold-change distribution of the DEG. C, Correlation analysis for S-RepA versus S-RepA ${ }^{\mathrm{UT}}$. The Pearson correlation coefficient was shown in the upper left corner of the plot. D, Comparison of DEG data and quantitative reverse-transcription polymerase chain reaction (qRT-PCR) results. "Concordant up" means that the DEG were determined to be upregulated for both the DGE and qRT-PCR analyses. "Concordant down" means that the DEG were downregulated in both DGE and qRT-PCR analyses. "Reverse exhibition" means that, for the DGE and qRT-PCR analyses, the relative changes in gene expression for the DEG were opposite. 
significant changes in these fluorescence parameters in $35 \mathrm{~S}$ RepA-infiltrated leaves at 18 hpi compared with the controls (Fig. 2B to E), which might be due to compensation of early photosynthetic products.

\section{Effects of RepA expression on chloroplast ultrastructure.}

Chloroplasts have the potential to act as delicate sensors of biotic stress, because they harbor numerous biological functions that are disrupted by environmental elicitors. Other than their role in photosynthesis, chloroplasts constitute a very important source of defense signaling molecules, including hormones and secondary messengers such as calcium and reactive oxygen species (ROS) (Serrano et al. 2016). Therefore, it was important to know whether the transient expression of RepA in $N$. benthamiana leaves had any influence on chloroplast ultrastructure. The leaf blades infiltrated with $35 \mathrm{~S}-\mathrm{RepA}$ or $35 \mathrm{~S}-$ $\mathrm{RepA}^{\mathrm{UT}}$ were collected at two time points: $18 \mathrm{hpi}$ (early stage, showing no symptom) and 72 hpi (late stage, showing sporadic necrosis). Ultrathin sections were prepared from both samples, followed by examination under the transmission electron microscope. We noticed that the number of chloroplasts had decreased in the 35S-RepA-infiltrated leaves at 72 hpi (Fig. 3A). Moreover, the chloroplasts appeared to be damaged, with the thylakoids in a chaotic arrangement, which was especially associated with a retardation in thylakoid formation and low numbers of grana and thylakoid lamellae (Fig. 3B). In contrast, clearly defined thylakoid layers, highly developed grana, dense ground substance lamellar structure, and ordered thylakoid lamellae were observed in leaves inoculated with the untranslatable mutant (35S-RepA ${ }^{\mathrm{UT}}$ ) (Fig. 3A and B). These data provided confirmation that the late expression of RepA caused disruptions to the chloroplast ultrastructure. However, our electron microscopic analysis of $N$. benthamiana leaves showed that the chloroplast ultrastructure appeared to be normal in the 35S-RepA-infiltrated leaves at 18 hpi (Fig. 3A and B), which was consistent with our analysis of chlorophyll fluorescence parameters.

Starch is one of the major products of photosynthesis in the leaves of land plants, and it can be classified into two types: (i) leaf starch located in photosynthetic organs and (ii) reserve starch located in storage organs (Wang et al. 2013). We used electron microscopy to determine the number of leaf starch granules in $N$. benthamiana for 35S-RepA versus 35S-RepA ${ }^{\mathrm{UT}}$. Consistent with a significant downregulation of photosynthesis during RepA-induced HR-type cell death, at $72 \mathrm{hpi}$, the number of chloroplast-localized starch granules was markedly reduced in leaves inoculated with $35 \mathrm{~S}-\mathrm{RepA}$ in comparison with the stable number observed in the healthy plants and the 35SRepA $^{\text {UT }}$ control (Fig. 3A).

\section{NbFDN1 in the electron transport chain was downregulated by $\operatorname{RepA}$ expression.}

The electron transport chain is considered to be an important center of photosynthesis. In plant chloroplasts, $\mathrm{Fd}$ participate in electron flow by receiving and donating electrons (Hanke et al. 2004). In our analysis of Illumina deep-sequencing data, we found that transcription of the ortholog of $\mathrm{Fd}-1$ from $N$. benthamiana (NbFDN1) was downregulated in the S-RepA versus S-RepA ${ }^{\mathrm{UT}}$ comparison. Further qRT-PCR analysis confirmed that the mRNA levels of $N b F D N 1$ were reduced by approximately twofold in the $35 \mathrm{~S}-\mathrm{RepA}$ inoculated leaves as compared with those inoculated with $35 \mathrm{~S}-\operatorname{RepA}^{\mathrm{UT}}$ at 18 or $36 \mathrm{hpi}$, and the mRNA levels decreased by more than fourfold at 72 hpi (Fig. 4A). To further determine whether NbFDN1 gene transcription was also affected by ODV infection, Agrobacterium tumefaciens carrying the ODV infectious clone was infiltrated into fully expanded leaves of $N$. benthamiana. Subsequent qRT-PCR assays showed that expression of NbFDN1 was reduced by approximately twofold when compared with the empty vector control at 3 days postinoculation (dpi) (Fig. 4B).

We then cloned and sequenced the full-length coding region of $N b F D N 1$ from the $N$. benthamiana cDNA library, and found that it encodes a predicted protein of 144 amino acids. A phylogenetic analysis revealed that the deduced amino acid sequence of NbFDN1 is closely related to predicted proteins from $N$. sylvestris, N. tabacum, Solanum lycopersicum, S. tuberosum, S .pennellii, and Capsicum annuum, with identities of $86.4,99.3,94.4,64.7,89.6,88.2$, and $82.6 \%$, respectively (Fig. 5A). As a typical characteristic of $\mathrm{Fd}$, sequence analysis identified an internal region within NbFDN1 that contains a conserved domain known as $2 \mathrm{Fe}-2 \mathrm{~S}$ (Fig. 5B). To determine the intracellular distribution pattern of NbFDN1, NbFDN1-fused in-frame to green fluorescent protein (GFP) (NbFDN1:GFP) was expressed in $N$. benthamiana protoplasts. Following agroinfiltration, laser confocal microscopy images showed that NbFDN1-GFP localizes to the surface of the chloroplast, whereas GFP alone was distributed uniformly throughout the cytoplasm in the control protoplasts (Fig. 5C). A further bimolecular fluorescence complementation (BiFC) assay revealed that NbFDN1 could self-interact in the chloroplast when NbFDN1 ORF was cloned into BiFC transformation

Table 1. Main Kyoto Encyclopedia of Genes and Genomes (KEGG) pathways enriched with differentially expressed genes (DEG) by Oat dwarf virus RepA expression

\begin{tabular}{|c|c|c|c|}
\hline Pathway & $\begin{array}{c}\text { Number of upregulated DEG/ } \\
\text { downregulated DEG }\end{array}$ & Genes per KEGG annotation (\%) & Pathway ID \\
\hline Metabolic pathways & $686 / 1,327$ & 25.55 & ko01100 \\
\hline Biosynthesis of secondary metabolites & $401 / 674$ & 13.65 & ko01110 \\
\hline Plant hormone signal transduction & $234 / 346$ & 7.36 & ko04075 \\
\hline Plant-pathogen interaction & $282 / 274$ & 7.06 & ko04626 \\
\hline Starch and sucrose metabolism & $86 / 154$ & 3.05 & ko00500 \\
\hline Phenylpropanoid biosynthesis & $117 / 96$ & 2.70 & ko00940 \\
\hline Protein processing in endoplasmic reticulum & $77 / 112$ & 2.40 & ko04141 \\
\hline Ribosome & $15 / 170$ & 2.35 & ko03010 \\
\hline RNA transport & $39 / 124$ & 2.07 & ko03013 \\
\hline $\begin{array}{l}\text { Amino sugar and nucleotide sugar } \\
\text { metabolism }\end{array}$ & $69 / 75$ & 1.83 & ko00520 \\
\hline Photosynthesis (light reaction) & $4 / 136$ & 1.78 & ko00195 \\
\hline Ubiquitin mediated proteolysis & $69 / 46$ & 1.46 & ko04120 \\
\hline $\begin{array}{l}\text { Carbon fixation in photosynthetic organisms } \\
\text { (containing Calvin cycle) }\end{array}$ & $20 / 87$ & 1.36 & ko00710 \\
\hline
\end{tabular}


vectors and subsequently agroinfiltrated in $N$. benthamiana leaves (Fig. 5D).

\section{NbFDN1 affects host photosynthesis and oxidative bursts.}

Because Fd are known to participate in electron flow, we chose to analyze the activities of Fv/Fm, Y(II), and ETR in NbFDN1-silenced and nonsilenced $N$. benthamiana plants obtained through VIGS using the TRV vector to investigate the function of NbFDN1 in host photosynthesis. The transcriptional level of NbFDN1 was reduced by more than $90 \%$ in silenced plants, which showed strong yellowing and mosaic symptoms in the upper leaves (Fig. 6A and B). Plants inoculated with TRV:GFP (as a control) showed slight crinkling of leaves early in the infection period that disappeared gradually (Fig. 6A and B). Our data show that significant reductions in Fv/Fm, Y(II), and ETR were observed in NbFDN1-silenced plants compared with the nonsilenced control plants (Fig. 6C to F). These findings provide evidence that photosynthetic-related processes are strongly affected by suppression of $\mathrm{NbFDN1}$ transcription.

Oxidative bursts or the generation of ROS such as $\mathrm{H}_{2} \mathrm{O}_{2}$ are regarded as an early plant immune signal response to biotic or abiotic stresses. Using the 3,3-diaminobenzidine (DAB)uptake method, we found that dark-brown areas were clearly visible in $N b F D N 1$-silenced leaves inoculated with mock but not in the nonsilenced controls (Fig. 6G). When NbFDN1silenced $N$. benthamiana plants were challenged with $35 \mathrm{~S}$ RepA, increased $\mathrm{H}_{2} \mathrm{O}_{2}$ production was observed compared with the nonsilenced control plants challenged with 35SRepA (Fig. 6G). These results indicate that downregulated expression of $N b F D N 1$ led to a significant increase in $\mathrm{H}_{2} \mathrm{O}_{2}$ accumulation.

Table 2. Expression profiles of partial differentially expressed genes by Oat dwarf virus RepA expression involved in the photosynthesis pathway

\begin{tabular}{|c|c|c|c|c|}
\hline Category or gene ID & Species & Homologous function (predicted) & Accession number & Fold change \\
\hline NbS00004889g0003.1 & Ricinus communis & ATP synthase $\gamma$ chain 2 & XP_002525795.1 & 8.29 \\
\hline NbS00005825g0012.1 & Lotus japonicus & Photosystem II (PSII) PsbW protein & AFK39129.1 & 3.98 \\
\hline Nbs00013207g0005.1 & Nicotiana sylvestris & Ferredoxin, root R-B1-like & XM_009806753.1 & 2.66 \\
\hline Nbs00022524g0005.1 & N. sylvestris & Cytochrome c6 & XM_009774828.1 & 1.08 \\
\hline Nbs00019305g0029.1 & N. sylvestris & Fruit protein pKIWI502 & XM_009782044.1 & -1.23 \\
\hline Nbs00018068g0003.1 & N. tabacum & Ferredoxin-NADP oxidoreductase, complete cds & AB004307.1 & -1.25 \\
\hline Nbs00029128g0009.1 & N. tabacum & Ferredoxin-NADP reductase & Y14032.1 & -2.07 \\
\hline Nbs00026206g0010.1 & N. tomentosiformis & PSII reaction center $\mathrm{W}$ protein & XM_009599623.1 & -2.89 \\
\hline Nbs00030360g0002.1 & N. sylvestris & Ferredoxin & XM_009798334.1 & -3.12 \\
\hline Nbs00029630g0003.1 & N. sylvestris & PSII $10-\mathrm{kDa}$ polypeptide & XM_009776249.1 & -3.49 \\
\hline Nbs00028467g0001.1 & N. tabacum & $\delta$ Subunit of chloroplast ATP synthase & X63607.1 & -3.61 \\
\hline Nbs00024956g0001.1 & N. sylvestris & ATP synthase $\delta$ chain, chloroplastic & XM_009775414.1 & -3.64 \\
\hline Nbs00008666g0006.1 & N. sylvestris & Photosystem I (PSI) subunit O-like & XM_009791082.1 & -3.75 \\
\hline Nbs00037361g0001.1 & N. sylvestris & PSI-D2 protein & X60008.1 & -4.13 \\
\hline Nbs00013563g0003.1 & N. benthamiana & Chloroplast PsbQ1 precursor (psbQ1) & JF897611.1 & -4.17 \\
\hline Nbs00020769g0006.1 & N. benthamiana & Chloroplast PSII 22-kDa component (psbS1) & EU645483.1 & -4.25 \\
\hline Nbs00030369g0003.1 & N. benthamiana & Chloroplast plastocyanin precursor & FJ598042.1 & -4.39 \\
\hline Nbs00038002g0008.1 & N. tomentosiformis & PSII reaction center $\mathrm{W}$ protein & XM_009614530.1 & -4.96 \\
\hline Nbs00005430g0008.1 & N. sylvestris & ATP synthase $\delta$ chain & XM_009782393.1 & -5.05 \\
\hline Nbs00010241g0007.1 & N. sylvestris & ATP synthase subunit $\mathrm{b}$ & XM_009776521.1 & -5.33 \\
\hline Nbs00019085g0006.1 & N. sylvestris & PSI reaction center subunit IV B & NM_001302598.1 & -5.38 \\
\hline Nbs00023165g0008.1 & N. tabacum & $\gamma$ Subunit of ATP synthase & X63606.1 & -5.5 \\
\hline Nbs00035687g0007.1 & N. tabacum & Precursor of PSII 22-kDa protein & X84225.1 & -5.56 \\
\hline Nbs00038205g0008.1 & N. sylvestris & PSII reaction center $\mathrm{W}$ protein & XM_009799309.1 & -5.61 \\
\hline Nbs00061418g0005.1 & N. tabacum & Chloroplast Rieske FeS precursor protein 2 & X66010.1 & -5.67 \\
\hline Nbs00010498g0007.1 & N. benthamiana & Chloroplast PsbQ2 precursor (psbQ2) & JF897612.1 & -5.77 \\
\hline Nbs00025048g0006.1 & N. tabacum & psaH gene for PSI psaH protein & X61665.1 & -5.99 \\
\hline Nbs00021386g0003.1 & N. tomentosiformis & PSI reaction center subunit psaK & XM_009603102.1 & -6.12 \\
\hline Nbs00012615g0018.1 & N. tomentosiformis & PSI subunit O-like & XM_009589516.1 & -6.17 \\
\hline Nbs00010560g0001.1 & N. sylvestris & Plastocyanin & XM_009773333.1 & -6.36 \\
\hline Nbs00028915g0014.1 & N. attenuata & PSI subunit XI & AF54 2546.1 & -6.57 \\
\hline Nbs00018155g0021.1 & N. sylvestris & PSI reaction center subunit VI-1 & NM_001302570.1 & -6.6 \\
\hline Nbs00028820g0007.1 & N. benthamiana & Chloroplast PsbP1 precursor (psbP1) & JF897607.1 & -6.61 \\
\hline Nbs00027134g0004.1 & N. benthamiana & Chloroplast PsbO4 precursor (psbO4) & JF897606.1 & -6.77 \\
\hline Nbs00002520g0008.1 & N. benthamiana & Chloroplast PsbP2 precursor (psbP2) & JF897608.1 & -7.04 \\
\hline Nbs00040113g0008.1 & N. sylvestris & PSI reaction center subunit VI-2 & XM_009786328.1 & -7.1 \\
\hline Nbs00010454g0009.1 & N. benthamiana & Chloroplast PsbO3 precursor (psbO3) & JF897605.1 & -7.19 \\
\hline Nbs00035792g0016.1 & N. sylvestris & PSI reaction center subunit III & XM_009779439.1 & -7.21 \\
\hline Nbs00002704g0011.1 & N. benthamiana & PSI reaction center subunit X PSI-K & AY899937.1 & -7.44 \\
\hline Nbs00032545g0003.1 & N. benthamiana & Chloroplast PsbO1 precursor (psbO1) & JF897603.1 & -7.52 \\
\hline Nbs00029078g0004.1 & N. sylvestris & PSI reaction center subunit $\mathrm{V}$ chloroplastic-like & XM_009778383.1 & -7.58 \\
\hline Nbs00010505g0002.1 & N. tabacum & PSI reaction center subunit $\mathrm{X}$ psaK & AY220078.1 & -7.73 \\
\hline Nbs00038432g0004.1 & N. sylvestris & PSI reaction center subunit IV A & NM_001302581.1 & -7.76 \\
\hline Nbs00019818g0001.1 & N. benthamiana & Chloroplast PsbO2 precursor (psbO2) & JF897604.1 & -7.78 \\
\hline Nbs00006619g0004.1 & N. sylvestris & PSII core complex proteins psbY & XM_009782664.1 & -7.93 \\
\hline Nbs00041800g0010.1 & N. tabacum & Putative $16-\mathrm{kDa}$ membrane protein & $\mathrm{AJ} 272037.1$ & -8.04 \\
\hline Nbs00007132g0019.1 & N. benthamiana & Chloroplast PsbP3 precursor (psbP3) & JF897609.1 & -8.19 \\
\hline Nbs00000058g0018.1 & N. tabacum & Putative PSI subunit III precursor & AY220077.1 & -8.37 \\
\hline Nbs00038282g0004.1 & N. tabacum & PSI reaction center subunit (PsaN) & GU187328.1 & -8.37 \\
\hline Nbs00010452g0020.1 & N. benthamiana & Chloroplast PsbP4 precursor (psbP4) & JF897610.1 & -9.27 \\
\hline Nbs00018180g0004.1 & N. tabacum & PSI reaction center subunit (PsaN) & GU187328.1 & -9.68 \\
\hline
\end{tabular}


NbFDN1 interacts with NbTsip1.

Tobacco Tsip1, a DnaJ-type Zn finger protein, was previously reported to directly interact with tobacco stress-induced gene 1 (Tsi1) and potentiates Tsi1-mediated transcriptional activation of stress-responsive genes (Ham et al. 2006). Our qRT-PCR analysis confirmed that the mRNA levels of NbTsip1 ( $N$. benthamiana Tsip1-like homolog) were increased 2.9-, 1.5-, and 1.5 -fold at 18,36 , and $72 \mathrm{hpi}$, respectively, after the leaves

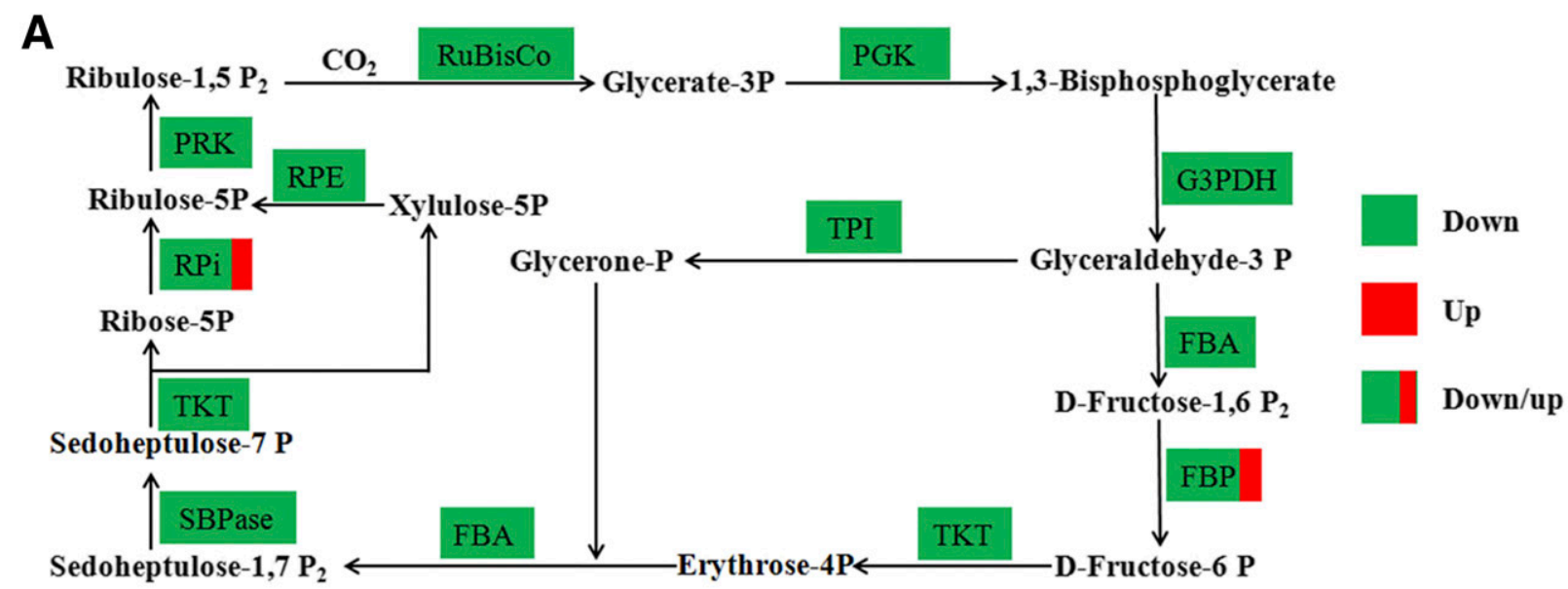

B
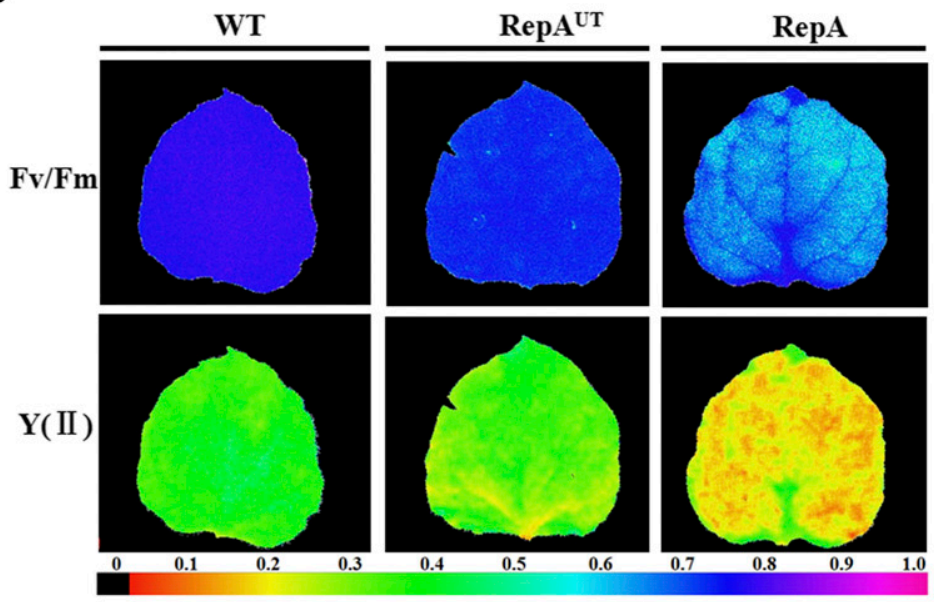

C



D

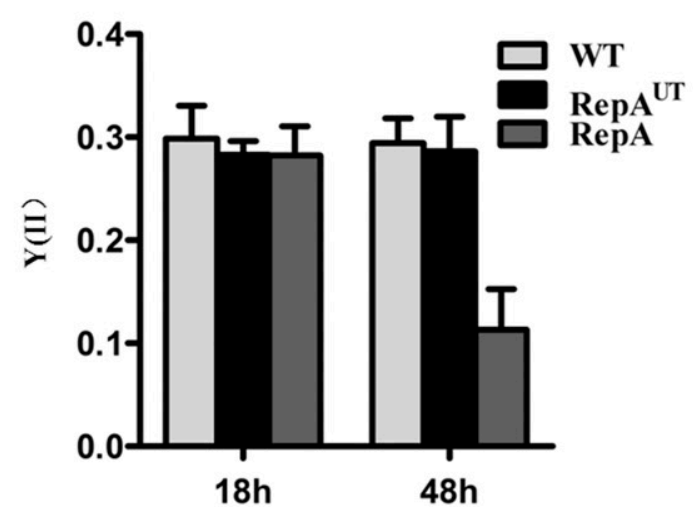

$\mathbf{E}$



Fig. 2. Effects of Oat dwarf virus RepA transient expression on photosynthesis. A, RepA transient expression impeded the Calvin-Benson cycle. Ribulase-1,5bisphosphate carboxylase oxygenase (RuBisCO), phosphoglycerate kinase (PGK), glyceraldehyde-3-phosphate dehydrogenase (G3PDH), triosephosphate isomerase (TPI), fructose-bisphosphate aldolase (FBA), fructose-1,6-bisphosphatase (FBP), transketolase (TKT), sedoheptulose bisphosphatase (SBPase), ribulose-phosphate 3-epimerase (RPE), ribose-5 phosphopentose isomerase (RPi), and phosphoribulokinase (PRK) were differentially regulated in this pathway. B, Images showing the maximum photochemical efficiency (Fv/Fm) and the actual photochemical efficiency (Y(II)) of photosystem II of whole leaves inoculated with agrobacterium containing $35 \mathrm{~S}-\mathrm{RepA}$ or $35-\mathrm{RepA} \mathrm{UT}^{\mathrm{UT}} 48 \mathrm{~h}$ postinoculation (hpi). WT $=$ wild type. The false color code depicted at the bottom of the image ranges from 0 (black) to 1.0 (purple). C, Average Fv/Fm values; D, average Y(II) values; and E, average electron transfer rate (ETR) values of whole leaves inoculated with agrobacterium containing $35 \mathrm{~S}-\mathrm{RepA}$ or $35-\mathrm{RepA}^{\mathrm{UT}}$ at 18 and 48 hpi. Bars represent the mean values, with standard errors of the mean, for one representative experiment $(n=10)$. 
were inoculated with 35S-RepA (Fig. 7A). Similarly, the expression of NbTsipl was elevated more than twofold in leaves inoculated with the ODV infectious viral clone when compared with the empty vector control at $3 \mathrm{dpi}$ (Fig. 7B).

Interestingly, BiFC assays demonstrated that the NbFDN1 protein directly interacts with NbTsip1 in the chloroplast (Fig. 8A). The complete ORF of NbFDN1 and NbTsip1 were cloned from the $N$. benthamiana cDNA library and inserted into BiFC transformation vectors. Following agroinfiltration, confocal microscopic images showed strong yellow fluorescent protein (YFP) fluorescence localized in the chloroplast at 48 dpi. No fluorescence was observed in cells coinfiltrated with the empty vectors $\mathrm{p} 2 \mathrm{YN}$ and $\mathrm{p} 2 \mathrm{YC}$ alone. We further confirmed that the interaction between NbFDN1 and NbTsip1 was specific using a yeast two-hybrid assay (Fig. 8B). We only observed growth for yeast cotransformed with both $N b F D N 1$ and NbTsip1 (AD-NbFDN1 + BD-NbTsip1 or AD-NbTsip1 + BD-NbFDN1).
We were interested to learn what happened after NbFDN1 directly interacted with NbTsip1. Consequently, our key observation was that there is a negative correlation between the transcription of $N b F D N 1$ and NbTsipl. qRT-PCR analysis showed that $N b T$ sipl-specific mRNA levels increased nearly threefold in NbFDN1-silenced plants (Fig. 8C).

\section{NbFDN1 and NbTsip1 are involved}

in the regulation of RepA-induced HR.

By silencing the NbFDN1 or NbTsipl genes using the TRVVIGS vector (Fig. 6A and B; Supplementary Fig. S5), we were able to investigate the function of NbFDN1 and NbTsip1 in the regulation of the RepA-induced HR-like response in $N$. benthamiana. A. tumefaciens carrying 35S-RepA was then infiltrated into the upper, fully expanded leaves of the NbFDN1- or NbTsipl-silenced plants. For $50 \%$ of the NbFDN1-silenced plants to develop the HR-type response, 3.5 dpi was required (Fig. 9A). By contrast, $50 \%$ of the control
A


B

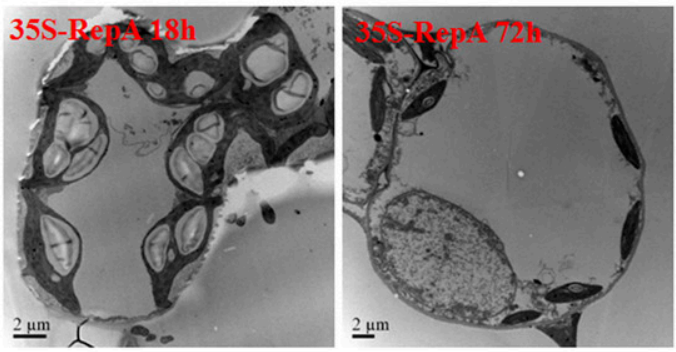

Fig. 3. Effects of Oat dwarf virus RepA transient expression on chloroplast ultrastructure. A, Electron microscopy of Nicotiana benthamiana leaf tissues collected from the wild type (WT) and plants inoculated with 35S-RepA or 35-RepA ${ }^{\mathrm{UT}}$. After staining with uranyl acetate and lead citrate, the sections were examined under an electron microscope. Bars $=2 \mu \mathrm{m}$. B, Electron microscopy of chloroplasts in the leaf tissues as described above. Bars $=0.5$ or $1 \mu \mathrm{m}$.

A

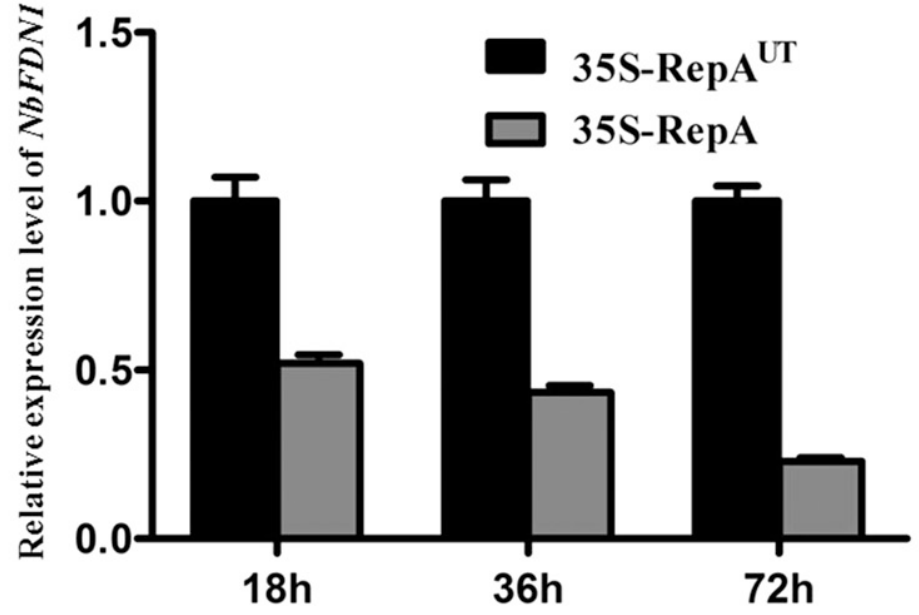

B

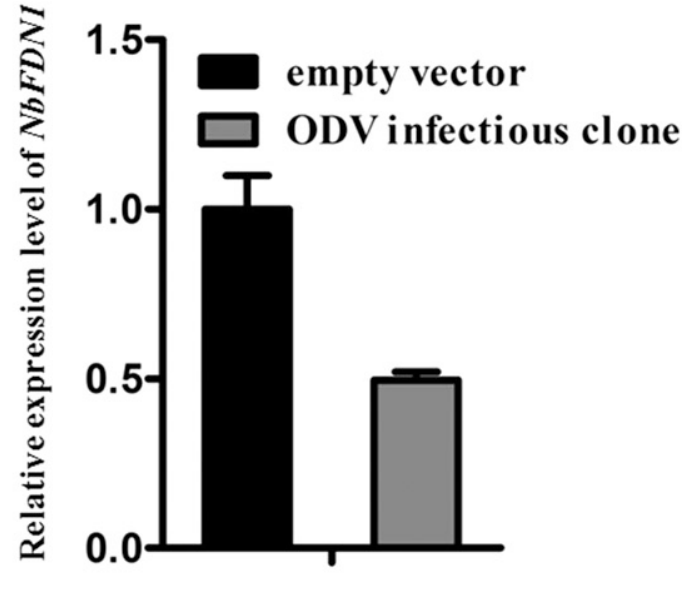

Fig. 4. Time course quantitative reverse-transcription polymerase chain reaction analysis of NbFDN1-specific mRNA expressed in Nicotiana benthamiana leaves inoculated with A, agrobacterium containing 35S-RepA or B, the Oat dwarf virus (ODV) infectious clone. The GADPH gene was used as an internal reference gene. Bars represent the mean values, with standard errors of the mean, for one representative experiment $(n=10)$. Relative mRNA levels of $N b F D N 1$ in leaves agroinfiltrated with $35 \mathrm{~S}-\mathrm{RepA}^{\mathrm{UT}}$ or the empty vector were arbitrarily set to 1 . 
plants required more than 5 dpi to show cell death, at which time $90 \%$ of the NbFDN1-silenced plants had developed HRtype cell death, which was shown to be significantly different by Student's $t$ test $(P<0.05)$. Interestingly, $50 \%$ of the
NbTsipl-silenced plants developed HR more than 5.5 days after infiltration (Fig. 9A), suggesting a moderately delayed RepA-induced cell death compared with the control plants $(P<0.05)$. We also observed an acceleration in cell death



B
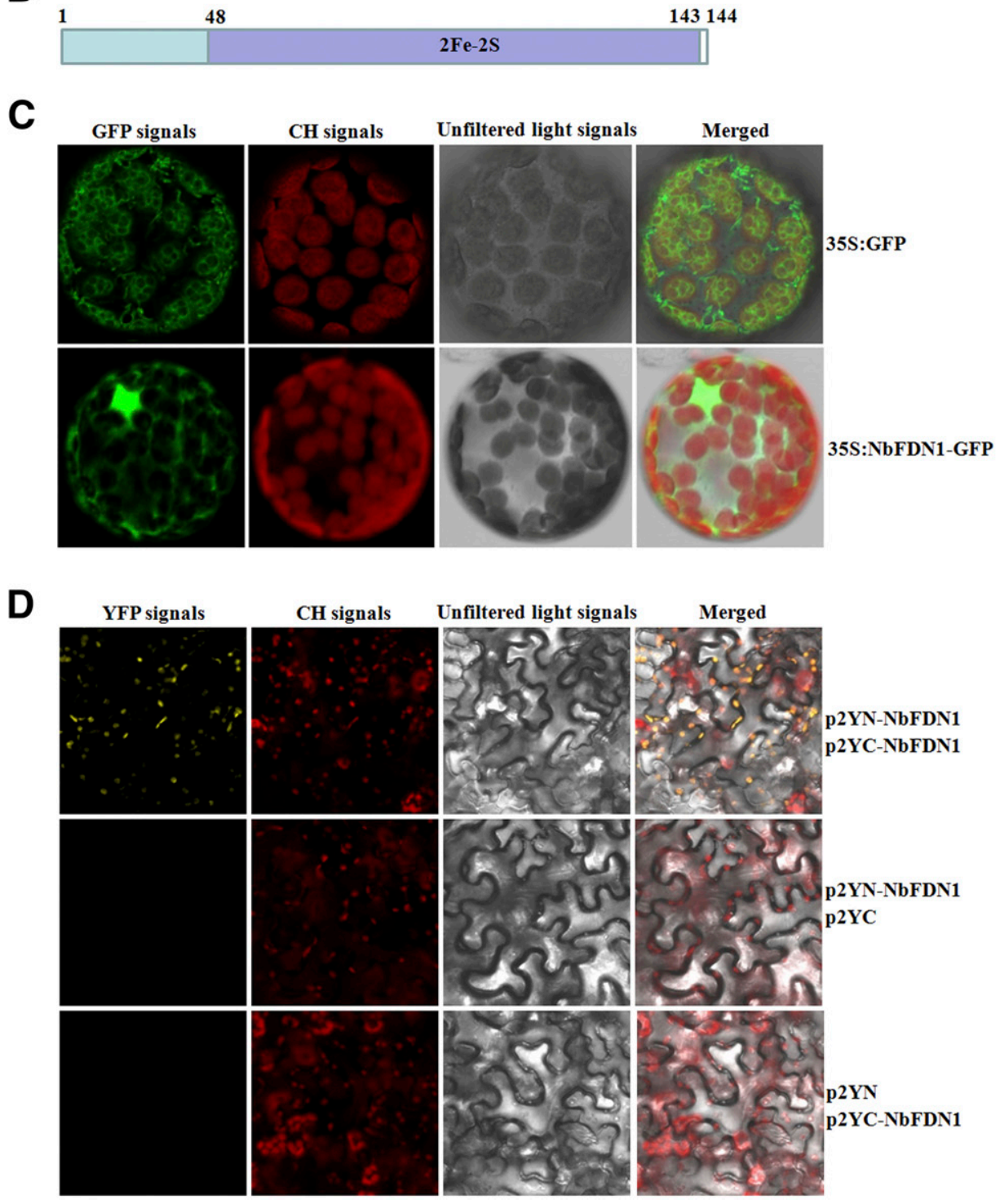

Fig. 5. Identification of NbFDN1 and localization of the protein to the surface of chloroplasts. A, Phylogenetic tree based on the deduced amino acid sequence of NbFDN1 analyzed with other plant ferredoxin sequences. B, Schematic representation of the genomic structure of NbFDN1 containing a domain conserved among the ferredoxin proteins. C, Subcellular localization of NbFDN1 fused to green fluorescent protein (GFP) in Nicotiana benthamiana protoplasts. D, Visualization of NbFDN1 self-interaction in $N$. benthamiana epidermal cells by bimolecular fluorescence complementation assay. YFP = yellow fluorescent protein. 
induced by ODV infection in NbFDN1-silenced $N$. benthamiana plants $(P<0.05)$ and a delay in cell death in NbTsipl-silenced $N$. benthamiana plants $(P<0.05)$ (Fig. 9B) compared with the control. Taken together, these results suggest that NbFDN1 and NbTsip1 play important roles in the development of HRtype cell death elicited by RepA. Further Western blot analysis showed no significant differences in accumulation levels of RepA proteins from NbFDN1-silenced or NbTsip1-silenced plants compared with those from the control plants (Fig. 9C), which ruled out the possibility that NbFDN1 and NbTsip1 were involved in modulating RepA-induced cell death through affecting RepA expression.
A

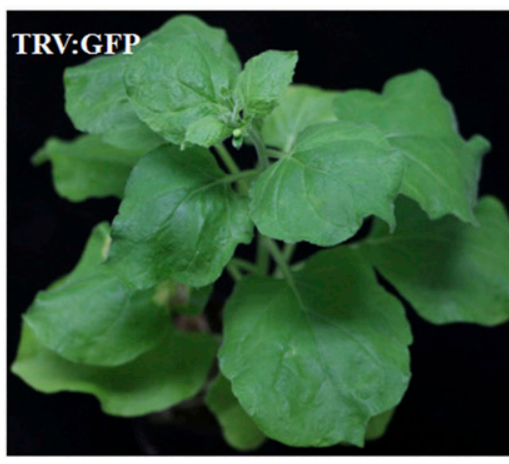

B

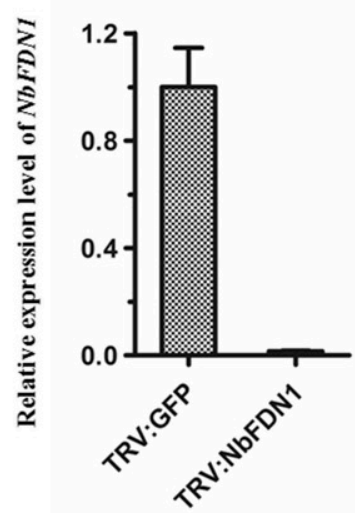

$\mathbf{F}$



C

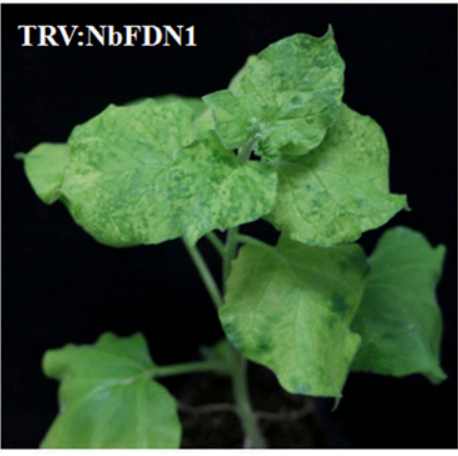

D



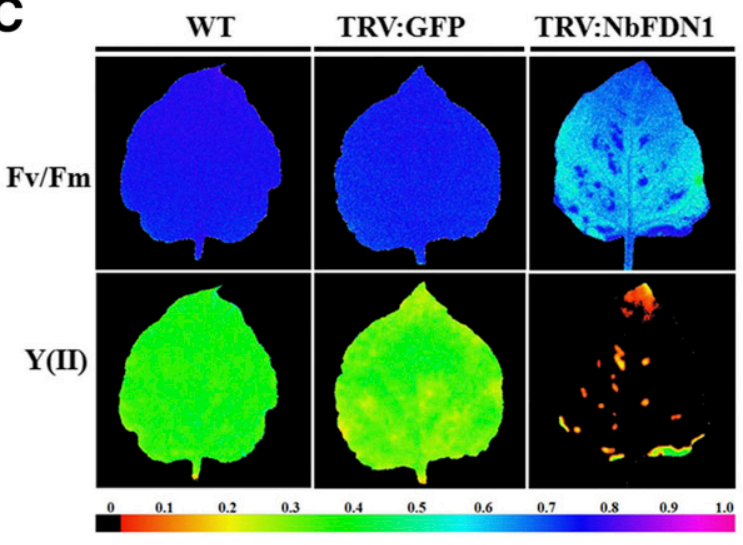

$\mathbf{E}$

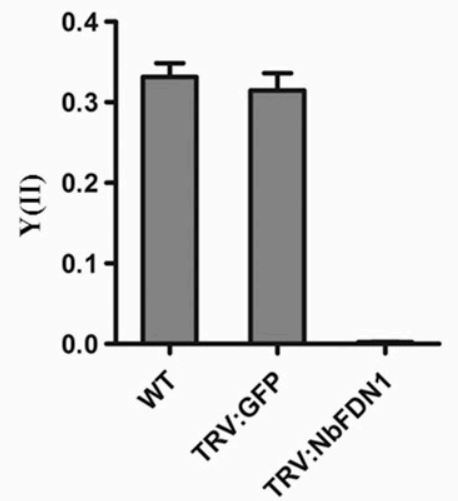

G
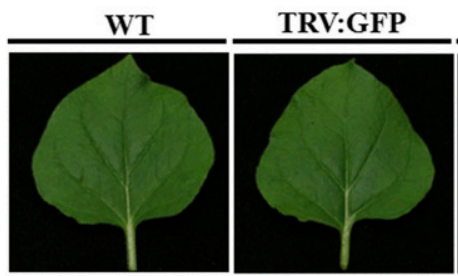

TRV:NbFDN1
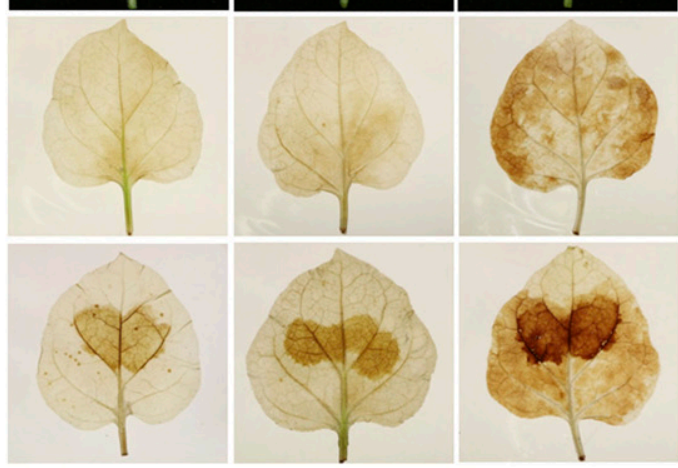

Mock inoculation

35S-RepA inoculation

Fig. 6. Effect of NbFDN1 gene silencing on photosynthesis rate and oxidative bursts. A, Symptoms associated with NbFDN1 silencing in Nicotiana benthamiana plants 10 days after agroinoculation with Tobacco rattle virus (TRV):NbFDN1. GFP = green fluorescent protein. B, Relative levels of $N b F D N 1$ specific mRNA in NbFDN1-silenced $N$. benthamiana plants. Error bars show standard deviations of 10 individual plants. C, Images of the maximum photochemical efficiency of photosystem II (PSII) (Fv/Fm), the actual photochemical efficiency of PSII (Y(II)), and the electron transfer rate (ETR) of whole leaves collected from $N b F D N 1$-silenced $N$. benthamiana plants. WT = wild type. Average $\mathbf{D}, \mathrm{Fv} / \mathrm{Fm} ; \mathbf{E}, \mathrm{Y}(\mathrm{II})$; and $\mathbf{F}$, ETR values of whole leaves collected from $N b F D N 1$-silenced $N$. benthamiana plants. Bars represent the mean values, with standard errors of the mean, for one representative experiment $(n=10)$. G, Images of 3,3-diaminobenzidine staining in mock-inoculated or 35S-RepA inoculated leaves at 2 days postinoculation. 


\section{DISCUSSION}

HR-related cell death has been extensively analyzed in various plant-virus interactions. Previously, it has been shown that some geminiviruses encode proteins that act as HR-type celldeath elicitors (Mubin et al. 2010; Sharma and Ikegami 2010). However, little is known about the molecular mechanisms associated with cell death induced by geminiviruses. In this study, we conducted a comparative analysis of gene expression profiles using high-throughput RNA-Seq technology to describe the effect of the ODV RepA protein on gene expression in $N$. benthamiana. One interesting aspect of our RNA-Seq analysis revealed that 7,878 significantly changed DEG mapped to 125 pathways, suggesting that comprehensive networks are involved in the regulation of RepA-induced cell death.

Among the DEG identified in our study, we focused our analysis on photosynthesis-related genes. The induction of genes involved in photosynthesis during stress conditions is well known in different plants. In contrast to the expected increase in photosynthesis in order to provide the energy for plant defense during pathogen infection, repression of photosynthesis is a frequent host response (Bilgin et al. 2010). Silencing two central photosynthetic proteins, RuBPCase and RuBPCase activase, in $N$. attenuata affected herbivore resistance mediated by the JA-Ile-dependent defense response and revealed the linkage between photosynthesis and biotic stress (Mitra and Baldwin 2008, 2014). Accordingly, in our study, a reduction in photosynthesis in $N$. benthamiana in response to RepA was detected at the transcriptional and physiological levels.

The chloroplast, in which photosynthesis takes place, is now considered to be a key defense organelle because it is able to perceive external signals that occur during biotic stresses (Serrano et al. 2016; Zhao et al. 2016). Previous reports based on optical coherence tomography indicated that changes in chloroplast structures are one of the earliest hallmarks in HR induced by harpin (an elicitor from the bacterium Erwinia amylovora) (Boccara et al. 2007). A recent report highlighted the importance of chloroplast functionality in the deregulation of plant cell death by affecting the interaction between Zymoseptoria tritici and wheat (Lee et al. 2015). Our studies have identified the destructive changes in chloroplast ultrastructure during transient expression of RepA, and suggest a linkage between damage to the photosynthetic apparatus and HR-like cell death. Previous reports have revealed many different chloroplast factors interacting with virus nucleic acids or proteins (Zhao et al. 2016), and it is plausible to presume that RepA proteins function through interaction with other chloroplast factors. Further experiments with plant-RepA interaction are necessary for elucidation of this aspect.

$\mathrm{Fd}$ contain a $[2 \mathrm{Fe}-2 \mathrm{~S}]$ cluster with a $\mathrm{CX} 4 \mathrm{CX} 2 \mathrm{CXnC}$-like pattern, which is an electron shuttle present in prokaryotes and algae (Hanke and Mulo 2013). The quantity of Fd found in plants is generally regulated by various biotic and abiotic stresses (Huang et al. 2007a; Mazouni et al. 2003; Zou et al. 2005). For example, the amount of Fd was reported to be upregulated by high light stress in Arabidopsis (Vorst et al. 1993) but downregulated following attacks by incompatible Pseudomonas syringae pv. glycinea, leading to HR-associated resistance in soybean (Zou et al. 2005). An intriguing finding suggests that disease resistance to bacterial pathogens can be affected by the amount of Fd-1 protein in plants (Huang et al. 2007a). Recently, although it is generally accepted that Fd are involved in the regulation of the plant resistance response (Huang et al. 2004, 2007b; Lin et al. 2010; Su et al. 2014; Tang et al. 2001), there have been conflicting results with respect to the roles played by Fd in HR-type cell death development.
Dayakar et al. (2003) reported that an Fd-like protein from sweet pepper intensified the ability of harpin $_{\mathrm{pss}}$, an elicitor from $P$. syringae, to induce the production of ROS, resulting in expression of the HR. In contrast, Zurbriggen et al. (2009) found that chloroplast active oxygen species build-up and local cell death were significantly reduced in Xanthomonas campestris pv. vesicatoria-inoculated tobacco plants expressing a cyanobacterial Fd-like protein.

Our RNA-Seq and qRT-PCR analyses showed that transcription of $N b F D N 1$ was significantly reduced after RepA expression. Using $N$. benthamiana plants silenced for $N b F D N 1$ expression, our results strongly suggested that chloroplastlocalized NbFDN1 is essential for RepA-induced HR-type cell death. Consistent with the findings of Zurbriggen et al. (2009), we showed that NbFDN1-silenced $N$. benthamiana plants have enhanced $\mathrm{H}_{2} \mathrm{O}_{2}$ production and activated host resistance, displaying typical HR-type lesions after RepA treatment. Interestingly, Ma et al. (2008) previously reported that a decrease in Fd-1 level was detected in TMV-infected tobacco plants, and that $N$. benthamiana plants in which the $F d-1$ gene was silenced accumulated $\mathrm{H}_{2} \mathrm{O}_{2}$ to a high level. These findings are consistent with our results, and seem to suggest that plants shift toward a downregulation of photosynthetic genes, with disruption of photosynthetic electron transfer and generation of ROS. ROS were identified as diffusible signal molecules, and the intensity and duration have been observed to vary in response to pathogen infection (Camejo et al. 2016). In some cases, the induced ROS might act as secondary messengers in the activation of signal transduction pathways. There is previous evidence to show that harpin ${ }_{\mathrm{pss}}$-induced cell death is significantly inhibited in the presence of diphenylene iodonium chloride and lanthanum chlorate, which are ROS inhibitors, suggesting that enhanced generation of ROS could be a possible cause of increased harpin $\mathrm{pss}_{\mathrm{s}}$-induced hypersensitive cell death (Dayakar et al. 2003). Taken together, we suspected that RepA may act upstream of NbFDN1 to negatively regulate $N b F D N 1$ gene expression, resulting in enhancing ROS generation and HR occurrence.

However, there are several lines of evidence to indicate that protein-protein interactions play an important role in the plant HR-like defense response (Thiel et al. 2012; Ueda et al. 2006). Using BiFC and yeast two-hybrid assays, we discovered that NbFDN1 interacts with Tsip1 in vivo. In view of the fact that the relative transcription of Tsipl was markedly increased in $N b F D N 1$-silenced plants, we can speculate that the interaction of NbFDN1 and Tsip1 has a negative effect on Tsipl gene expression. In our study, consistent with previous results showing that transgenic plants simultaneously coexpressing
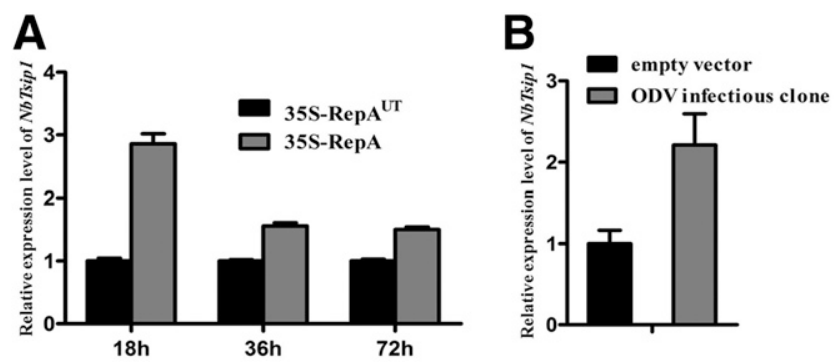

Fig. 7. Time course quantitative reverse-transcription polymerase chain reaction analysis of NbTsipl-specific mRNA expressed in Nicotiana benthamiana leaves inoculated with A, agrobacterium containing 35S-RepA or B, the Oat dwarf virus (ODV) infectious clone. The GADPH gene was used as an internal reference gene. Bars represent the mean values, with standard errors of the mean, for one representative experiment $(n=10)$. Relative mRNA levels of NbTsipl in leaves agroinfiltrated with $35 \mathrm{~S}-\mathrm{RepA}^{\mathrm{UT}}$ or the empty vector were arbitrarily set to 1 . 
Tsi1 and Tsip1 displayed stronger pathogen resistance and salt tolerance (Park et al. 2001), Tsip1-silenced plants showed a delay in RepA-induced HR. Previous research has shown that Tsip1 interacts directly with Tsi1, resulting in Tsip1 recruiting Tsi1 to the promoters of stress-related genes to potentiate Tsi1- mediated transcriptional activation (Ham et al. 2006). It might be reasonable to speculate that an additional pathway exists in which NbFDN1 has an effect on RepA-induced HR by indirectly modulating Tsi1 transcription activity to promote defenserelated gene expression.
A

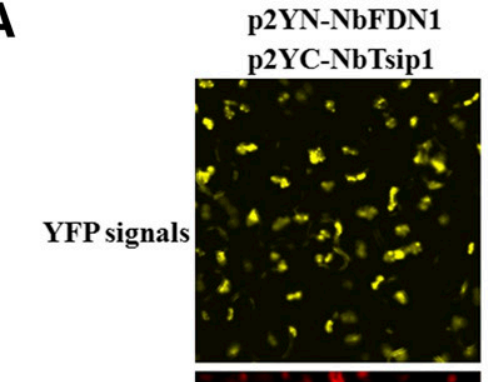

p2YN-NbFDN1
p2YC-NbTsip1

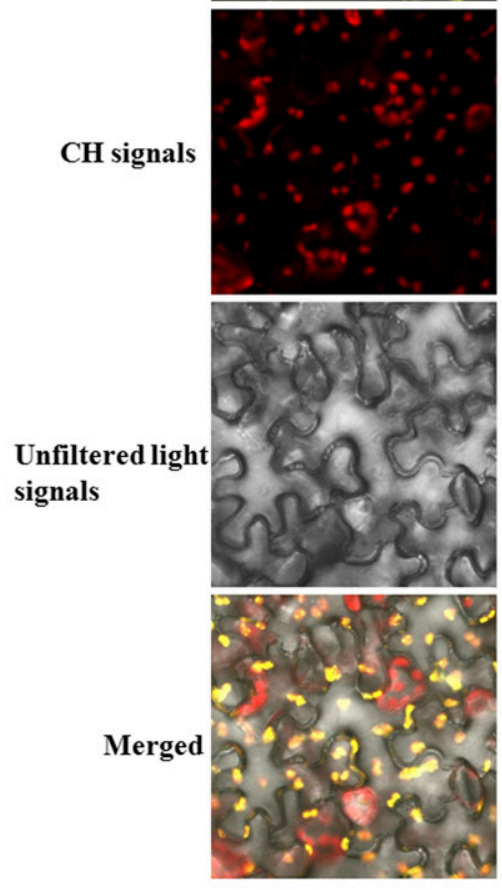

Unfiltered light
signals

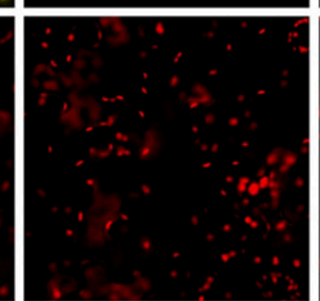

p2YN-NbFDN1

p2YC

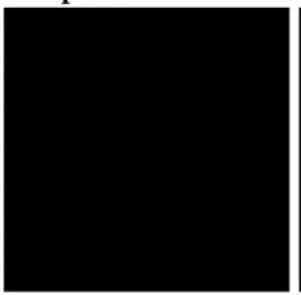

\section{p2YC-NbTsip1}
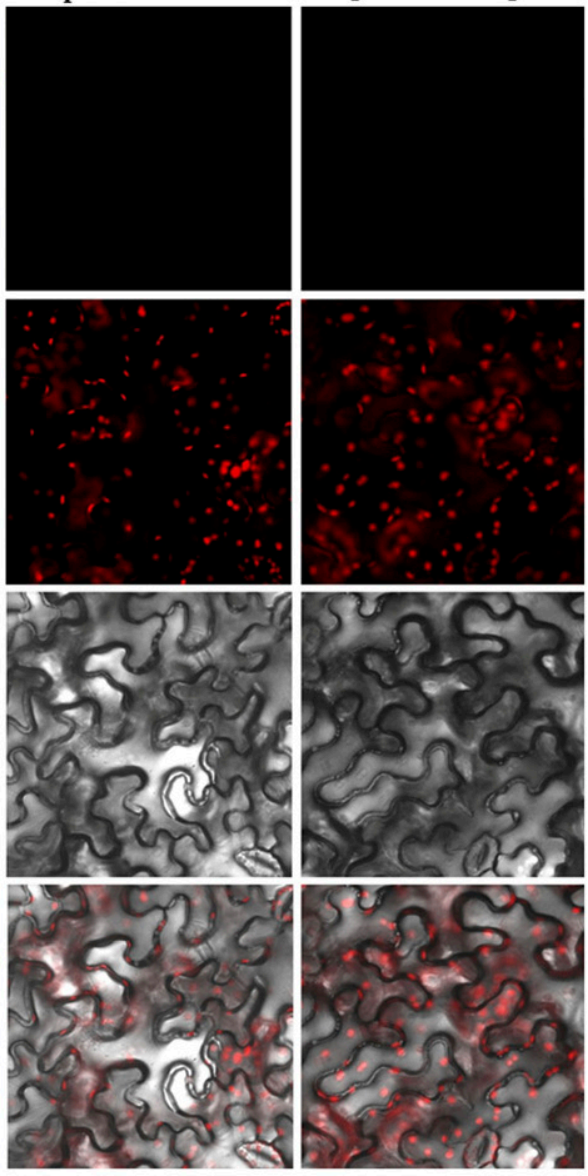

B

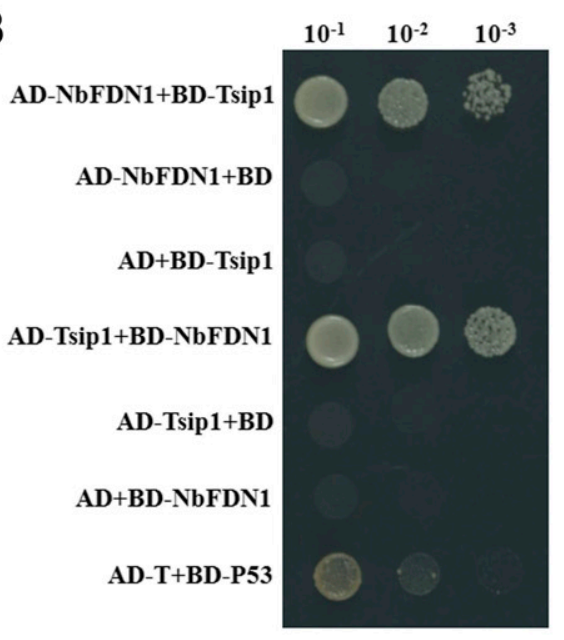

C



Fig. 8. Interaction of NbFDN1 and NbTsip1 in vivo. A, Bimolecular fluorescence complementation visualization of interactions between NbFDN1 and NbTsip1 in agroinfiltrated Nicotiana benthamiana leaves. YFP = yellow fluorescent protein and $\mathrm{CH}=$ red autofluoresce from chloroplast. B, Yeast two-hybrid assay of the NbFDN1 and NbTsip1 interaction in yeast strain Gold cotransformed with the plasmids shown on the left side of the panel and grown on an $\mathrm{SD} / \mathrm{His} /$-Leu/-Trp medium at three different concentrations. C, Quantitative reverse-transcription polymerase chain reaction analysis of NbTsipl transcripts expressed in leaves of NbFDN1-silenced $N$. benthamiana plants. The relative mRNA level of NbTsipl in leaves agroinfiltrated with Tobacco rattle virus green fluorescent protein (TRV:GFP) was arbitrarily set to 1 . 


\section{MATERIALS AND METHODS}

\section{Expression constructs.}

The expression constructs $35 \mathrm{~S}-\mathrm{RepA}$ and $35 \mathrm{~S}-\mathrm{RepA}^{\mathrm{UT}}$, containing an untranslatable RepA mutant and introduced an in-frame stop codon immediately downstream of the RepA start codon, were from a previous source (Qian et al. 2016).

\section{Agroinoculation of plants.}

All expression constructs were transformed into A. tumefaciens strain C58C1 through electroporation. Strains were incubated in yeast extract-peptone medium at $28^{\circ} \mathrm{C}$ overnight, then suspended in induction medium $(10 \mathrm{mM}$ morpholineethanesulfonic acid [MES], pH 5.7; $10 \mathrm{mM} \mathrm{MgCl}_{2}$; and $200 \mathrm{mM}$ acetosyringone) to an adjusted optical density at $600 \mathrm{~nm}$ $\left(\mathrm{OD}_{600}\right)$ of 1.0. The agrobacterium cultures were inoculated into leaves of $N$.benthamiana plants at the four-leaf stage using a $1-\mathrm{ml}$ syringe without a needle. The inoculated plants were grown in a greenhouse at $25^{\circ} \mathrm{C}$ under a photoperiod $16 \mathrm{~h}$ of light and $8 \mathrm{~h}$ of darkness per day.

\section{Sample preparation and library preparation.}

The agroinfiltrated leaves (1-g samples) were collected from $35 \mathrm{~S}-\mathrm{RepA}$ - or $35 \mathrm{~S}-\mathrm{RepA}^{\mathrm{UT}}$-inoculated $N$. benthamiana plants at $3 \mathrm{dpi}$. To reduce the experimental variation, each collected sample consisted of leaves from 10 different plants. The samples then were ground in liquid nitrogen and mixed with $10 \mathrm{ml}$ of TRIzol reagent (Invitrogen, Carlsbad, CA, USA). Total RNA was extracted and the concentration and purity were determined using a NanoDrop 2000 spectrophotometer (Thermo Scientific, Wilmington, DE, USA). The cDNA libraries were constructed in parallel using an Illumina kit following the manufacturer's instructions. Briefly, the mRNA was enriched from the total RNA using oligo(dT) magnetic beads. Following purification, the mRNA was then fragmented into short pieces (approximately $200 \mathrm{bp}$ ). First-strand cDNA was synthesized using RT and random hexamer primers, and the second-strand cDNA was synthesized using RNase $\mathrm{H}$ and DNA polymerase I. The double-stranded cDNA was then purified, and this was followed by an end repair process and ligation of sequencing adaptors. After the products were enriched by PCR amplification to create the cDNA library, the Agilent 2100 Bioanaylzer and the ABI StepOnePlus Real-Time PCR System were used to qualify and quantify the sample libraries for RNA-Seq.

\section{Illumina sequencing and data analysis.}

The two cDNA libraries from the 35S-RepA- or 35S$\mathrm{RepA}^{\mathrm{UT}}$-inoculated samples were sequenced in parallel using the Illumina Hiseq2000 sequencing platform (BGI, Shenzhen, China). The raw reads from the images were generated with the Solexa GA pipeline 1.6. After removal of low-quality reads, the processed reads were assembled using the Short Oligonucleotide Analysis Package (SOAP) de novo software and clustered

Fig. 9. Effect of $N b F D N 1$ and $N b T$ sip1 gene silencing on Oat dwarf virus (ODV) RepA-induced hypersensitive response (HR). Data show the percentage of Nicotiana benthamiana leaves exhibiting the HR when inoculated with agrobacterium containing either A, 35S-RepA or B, the ODV infectious clone into NbFDN1-silenced or NbTsipl-silenced plants. Data were derived from at least 10 plants in each treatment. Plants infected with Tobacco rattle virus green fluorescent protein (TRV:GFP) or the empty vector were used as controls. Error bars represent the standard deviations of three independent experiments. C, Western blot analysis of RepA accumulation in NbFDN1-silenced or NbTsip1-silenced plants inoculated with agrobacterium containing 35S-RepA using a RepA-specific antibody. Plants infected with TRV:GFP were used as controls. A probe against the housekeeping gene actin was used as a loading control. using the TIGR Gene Indices clustering tool. Clean reads were mapped to reference sequences or a reference gene set using SOAP aligner/SOAP2. No more than two mismatches were allowed in the alignments. The assembled unigenes larger than $350 \mathrm{bp}$ were analyzed by searching the GenBank and Swissprot databases using the BLASTX algorithm. GO annotations and COG classification of the unigenes were determined with Blast2go and Inter-ProScan software.

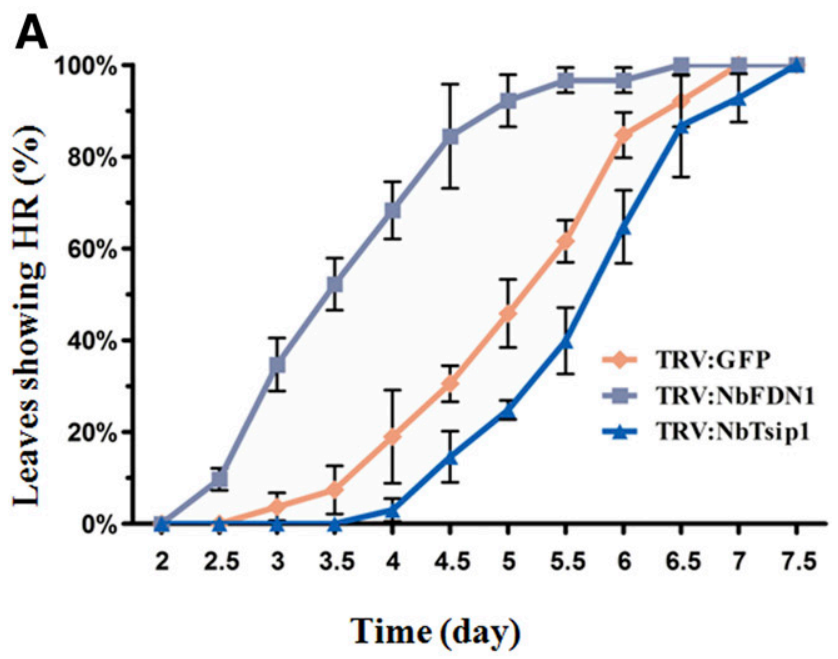

B

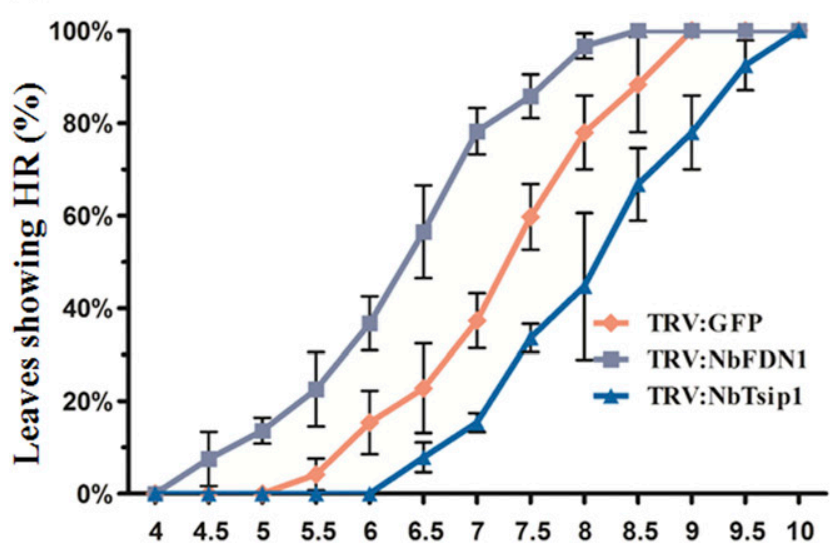

Time (day)

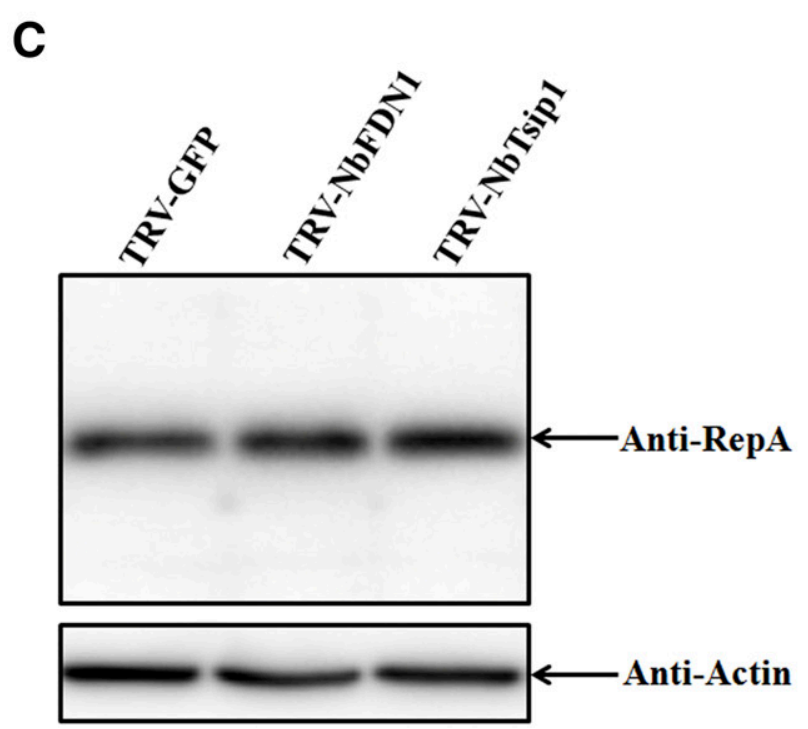




\section{Analysis of differential gene expression.}

A strict, rigorous algorithm was developed to identify DEG between the two different cDNA libraries. The FDR method was used to determine the $P$ value threshold in multiple tests and analyses. We used an FDR of 0.001 and an absolute value of $\log _{2}$ ratio $\geq 1$ as the threshold to judge the significance of differences in gene expression. The correlation between the two libraries was statistically assessed by calculation of the Pearson correlation coefficient.

\section{Pathway analysis and}

\section{KEGG pathway enrichment analysis of the DEG.}

All DEG were mapped to terms in the KEGG database, which is the major public pathway-related database, based on parameters (-p bl astx -e 1e-5 -m 8).

\section{GO functional classification.}

With nr annotation, we used the Blast2GO program, a widely recognized GO annotation software, to obtain GO annotation of the DEG, then used WEGO (GO functional classification) software to perform GO functional classification of the DEG and to understand the distribution of gene functions of the species at the macro level.

\section{qRT-PCR analysis.}

For qRT-PCR analysis, total RNA was extracted from the leaf samples using Trizol reagent (Invitrogen). Isolated RNA was reverse transcribed to generate cDNA using the ReverTra Ace qRT-PCR RT Master Mix with gDNA Remover kit (Toyobo Co. Ltd., Osaka, Japan) with oligo-dT primers, according to the manufacturer's instructions. qRT-PCR was carried out using the LightCycler 480 SYBR I Master kit (Roche Diagnostics Ltd., Rotkreuz, Switzerland) under the following conditions: $95^{\circ} \mathrm{C}$ for $5 \mathrm{~min} ; 40$ cycles of $95^{\circ} \mathrm{C}$ for $10 \mathrm{~s}, 60^{\circ} \mathrm{C}$ for $15 \mathrm{~s}$, and $72^{\circ} \mathrm{C}$ for $20 \mathrm{~s}$; followed by melting curve generation $\left(68\right.$ to $\left.95^{\circ} \mathrm{C}\right)$. The $G A D P H$ gene was used as the internal control for gene expression normalization.

\section{VIGS assays.}

For the VIGS assays, partial DNA fragments specific for the NbFDN1 (226 bp) or NbTsipl (450 bp) genes were amplified from $N$. benthamiana cDNA using gene-specific primer pairs (Supplementary Table S5) and introduced into the TRV2 VIGS vector as described by Liu et al. (2002) at the Xba1/BamH1 restriction enzyme sites. The recombinant plasmids were then introduced into A. tumefaciens strain EHA105 by electroporation. Agrobacterium strains harboring the VIGS vectors or empty pTRV2 were suspended in infiltration buffer $(10 \mathrm{mM}$ $\mathrm{MgCl}_{2}, 10 \mathrm{mM}$ MES, and $200 \mathrm{mM}$ acetosyringone) to an adjusted $\mathrm{OD}_{600}$ of 1.0 and mixed at a $1: 1$ ratio with agrobacterium cells carrying pTRV1. After 2 to $3 \mathrm{~h}$ of incubation at room temperature, the mixed agrobacterium cultures were infiltrated into the leaves of $N$. benthamiana plants. The VIGS efficiency was evaluated by comparing relative mRNA abundance in silenced plants with that in control plants using qRT-PCR analysis.

\section{Transmission electron microscopy.}

Infiltrated leaf discs were cut into small pieces (approximately 1 by $4 \mathrm{~mm}$ ) and fixed with $2.5 \%$ glutaraldehyde in $100 \mathrm{mM}$ phosphate-buffered saline (PBS; $\mathrm{pH}$ 7.0) for more than $4 \mathrm{~h}$ at $4{ }^{\circ} \mathrm{C}$, then refixed with $1 \% \mathrm{OsO}_{4}$ in PBS buffer $(100 \mathrm{mM}$, $\mathrm{pH}$ 7.0) at room temperature for $2 \mathrm{~h}$, and rinsed three times (15 min each) with PBS buffer. The fixed tissues were dehydrated in a graded ethanol series for $15 \mathrm{~min}$ each, then placed into absolute acetone for $20 \mathrm{~min}$. The sample tissues were embedded in mixtures of acetone and Spurr resin overnight.
Ultrathin sections $(70 \mathrm{~nm})$ were cut from the embedded tissues using the Ultracut E Ultramicrotome (Reichart-Jung, Vienna, Austria). The thin sections were stained with uranyl acetate and alkaline lead citrate for $15 \mathrm{~min}$ each. The stained sections were then observed under an electron microscope (JEOL model JEM-1230).

\section{BiFC assay.}

To construct the BiFC vectors, the full-length coding sequences of NbFDN1 and NbTsip1 were PCR amplified using the primer pairs BiFC-NbFDN1-F/BiFC-NbFDN1-R and BiFC-NbTsip1-F/BiFC-NbTsip1-R from an N. benthamiana leaf cDNA library and cloned into BiFC transformation vectors to produce the constructs p2YN-NbFDN1, p2YC-NbFDN1, p2YN-NbTsip1, and p2YC-NbTsip1. The plasmids were then individually introduced into A. tumefaciens strain C58C1 through electroporation. Emission of YFP fluorescence was detected in $N$. benthamiana leaves, and the cells were imaged using a confocal microscope (Leica TCS SP5, Mannheim, Germany) at $48 \mathrm{hpi}$.

\section{Yeast two-hybrid assays.}

The full-length coding sequences of NbFDN1 and NbTsip1 were PCR amplified and cloned into the AD/BD vector to produce the constructs AD-NbFDN1, BD-Tsip1, AD-Tsip1, and BD-NbFDN1. Yeast two-hybrid assays were performed essentially as described previously (Shen et al. 2011).

\section{Subcellular localization assays.}

The full coding sequence of NbFDN1 was PCR amplified with the primer pair F3-NbFDN1-F/F3-NbFDN1-R and fused to the $\mathrm{N}$ terminus of GFP by insertion into the pCHF3GFP vector. The recombinant plasmid was transformed into A. tumefaciens strain C58C1. Subcellular localization assays were performed as described previously (Kong et al. 2014). At $36 \mathrm{hpi}$, the released protoplasts were collected and examined under a confocal microscope for GFP fluorescence.

\section{Protein extraction and Western blots.}

Proteins were extracted from the leaves of $N$. benthamiana plants and probed with a rabbit anti-RepA polyclonal antibody, as previously described (Qian et al. 2016).

\section{Detection of oxidative bursts in plants.}

$\mathrm{H}_{2} \mathrm{O}_{2}$ was visually detected in the leaves of $N$. benthamiana plants by staining with DAB (Qian et al. 2016; ThordalChristensen et al. 1997).

\section{Measurements of photosynthetic parameters.}

Approximately 7 days after agroinfiltration, $N$. benthamiana plants were dark adapted for $30 \mathrm{~min}$, and chlorophyll fluorescence was then determined in the third to fourth leaf of each plant by Imaging PAM (IMAG-MAXI; Heinz Walz, Effeltrich, Germany). Minimal fluorescence yield (F0) was measured under a weak modulating light intensity of $125 \mu \mathrm{mol} \mathrm{m} \mathrm{m}^{-2} \mathrm{~s}^{-1}$, and maximal fluorescence yield (Fm) was measured with a saturating flash $\left(4,000 \mu \mathrm{mol} \mathrm{m} \mathrm{m}^{-2} \mathrm{~s}^{-1}\right)$ of light in a $0.8-\mathrm{s}$ pulse. Fv is the difference between $\mathrm{Fm}$ and F0. Fv/Fm $=(\mathrm{Fm}-\mathrm{F} 0) / \mathrm{Fm}$, $\mathrm{YII}=\left(\mathrm{F}^{\prime} \mathrm{m}-\mathrm{Fs}\right) / \mathrm{F}^{\prime} \mathrm{m} ; \mathrm{F}^{\prime} \mathrm{m}$ and $\mathrm{Fs}$ represent the maximal and steady-state fluorescence yields in a light-adapted state, respectively. $\mathrm{ETR}=0.84 \times \mathrm{PAR} \times \mathrm{YII} \times 0.5$, where PAR is the photosynthetically active radiation.

\section{ACKNOWLEDGMENTS}

We thank J. Schubert at the Federal Research Institute for Cultivated Plants, Germany, for kindly providing the infectious clone ODV-Hak14. 


\section{LITERATURE CITED}

Angel, C. A., and Schoelz, J. E. 2013. A survey of resistance to Tomato bushy stunt virus in the genus Nicotiana reveals that the hypersensitive response is triggered by one of three different viral proteins. Mol. PlantMicrobe Interact. 26:240-248.

Bilgin, D. D., Zavala, J. A., Zhu, J., Clough, S. J., Ort, D. R., and DeLucia, E. H. 2010. Biotic stress globally downregulates photosynthesis genes. Plant Cell Environ. 33:1597-1613.

Boccara, M., Schwartz, W., Guiot, E., Vidal, G., De Paepe, R., Dubois, A., and Boccara, A. C. 2007. Early chloroplastic alterations analysed by optical coherence tomography during a harpin-induced hypersensitive response. Plant J. 50:338-346.

Bozsó, Z., Maunoury, N., Szatmari, A., Mergaert, P., Ott, P. G., Zsíros, L. R., Szabó, E., Kondorosi, E., and Klement, Z. 2009. Transcriptome analysis of a bacterially induced basal and hypersensitive response of Medicago truncatula. Plant Mol. Biol. 70:627-646.

Brading, P. A., Hammond-Kosack, K. E., Parr, A., and Jones, J. D. 2000. Salicylic acid is not required for Cf-2- and Cf-9-dependent resistance of tomato to Cladosporium fulvum. Plant J. 23:305-318.

Camejo, D., Guzmán-Cedeño, Á., and Moreno, A. 2016. Reactive oxygen species, essential molecules, during plant-pathogen interactions. Plant Physiol. Biochem. 103:10-23.

Chen, H., Adam Arsovski, A., Yu, K., and Wang, A. 2017. Deep sequencing leads to the identification of eukaryotic translation initiation factor $5 \mathrm{~A}$ as a key element in Rsv1-mediated lethal systemic hypersensitive response to Soybean mosaic virus infection in soybean. Mol. Plant Pathol. 18: 391-404.

Collin, S., Fernández-Lobato, M., Gooding, P. S., Mullineaux, P. M., and Fenoll, C. 1996. The two nonstructural proteins from wheat dwarf virus involved in viral gene expression and replication are retinoblastomabinding proteins. Virology 219:324-329.

Dayakar, B. V., Lin, H. J., Chen, C. H., Ger, M. J., Lee, B. H., Pai, C. H., Chow, D., Huang, H. E., Hwang, S. Y., Chung, M. C., and Feng, T. Y. 2003. Ferredoxin from sweet pepper (Capsicum annuum L.) intensifying harpin $_{\mathrm{pss}}-$ mediated hypersensitive response shows an enhanced production of active oxygen species (AOS). Plant Mol. Biol. 51:913-924.

Geng, C., Wang, H. Y., Liu, J., Yan, Z. Y., Tian, Y. P., Yuan, X. F., Gao, R. and Li, X. D. 2017. Transcriptomic changes in Nicotiana benthamiana plants inoculated with the wild-type or an attenuated mutant of Tobacco vein banding mosaic virus. Mol. Plant Pathol. 18:1175-1188.

Ham, B. K., Park, J. M., Lee, S. B., Kim, M. J., Lee, I. J., Kim, K. J., Kwon, C. S., and Paek, K. H. 2006. Tobacco Tsip1, a DnaJ-type Zn finger protein, is recruited to and potentiates Tsi1-mediated transcriptional activation. Plant Cell 18:2005-2020.

Hanke, G. T., Kimata-Ariga, Y., Taniguchi, I., and Hase, T. 2004. A post genomic characterization of Arabidopsis ferredoxins. Plant Physiol. 134: 255-264.

Hanke, G., and Mulo, P. 2013. Plant type ferredoxins and ferredoxindependent metabolism. Plant Cell Environ. 36:1071-1084.

Hu, Z., Zhang, T., Yao, M., Feng, Z., Miriam, K., Wu, J., Zhou, X., and Tao, X. 2012. The 2 a protein of Cucumber mosaic virus induces a hypersensitive response in cowpea independently of its replicase activity Virus Res. 170:169-173.

Huang, H. E., Ger, M. J., Chen, C. Y., Pandey, A. K., Yip, M. K., Chou, H. W., and Feng, T. Y. 2007a. Disease resistance to bacterial pathogens affected by the amount of ferredoxin-I protein in plants. Mol. Plant Pathol. 8:129-137.

Huang, H. E., Ger, M. J., Yip, M. K., Chen, C. Y., Pandey, A. K., and Feng, T. Y. 2004. A hypersensitive response was induced by virulent bacteria in transgenic tobacco plants overexpressing a plant ferredoxin-like protein (PFLP). Physiol. Mol. Plant Pathol. 64:103-110.

Huang, H. E., Liu, C. A., Lee, M. J., Kuo, C. G., Chen, H. M., Ger, M. J. Tsai, Y. C., Chen, Y. R., Lin, M. K., and Feng, T. Y. 2007b. Resistance enhancement of transgenic tomato to bacterial pathogens by the heterologous expression of sweet pepper ferredoxin-I protein. Phytopathology 97:900-906.

Kawahara, Y., Oono, Y., Kanamori, H., Matsumoto, T., Itoh, T., and Minami, E. 2012. Simultaneous RNA-seq analysis of a mixed transcriptome of rice and blast fungus interaction. PLoS One 7:e49423.

Kim, B., Masuta, C., Matsuura, H., Takahashi, H., and Inukai, T. 2008. Veinal necrosis induced by turnip mosaic virus infection in Arabidopsis is a form of defense response accompanying HR-like cell death. Mol. Plant-Microbe Interact. 21:260-268.

Kim, B. M., Suehiro, N., Natsuaki, T., Inukai, T., and Masuta, C. 2010. The P3 protein of Turnip mosaic virus can alone induce hypersensitive response-like cell death in Arabidopsis thaliana carrying TuNI. Mol Plant-Microbe Interact. 23:144-152.
Kong, L., Wu, J., Lu, L., Xu, Y., and Zhou, X. 2014. Interaction between Rice stripe virus disease-specific protein and host PsbP enhances virus symptoms. Mol. Plant 7:691-708.

Lee, W. S., Devonshire, B. J., Hammond-Kosack, K. E., Rudd, J. J., and Kanyuka, K. 2015. Deregulation of plant cell death through disruption of chloroplast functionality affects asexual sporulation of Zymoseptoria tritici on wheat. Mol. Plant-Microbe Interact. 28:590-604.

Lin, Y. H., Huang, H. E., Wu, F. S., Ger, M. J., Liao, P. L., Chen, Y. R., Tzeng, K. C., and Feng, T. Y. 2010. Plant ferredoxin-like protein (PFLP) outside chloroplast in Arabidopsis enhances disease resistance against bacterial pathogens. Plant Sci. 179:450-458.

Liu, Y., Schiff, M., and Dinesh-Kumar, S. P. 2002. Virus-induced gene silencing in tomato. Plant J. 31:777-786.

Luque, A., Sanz-Burgos, A. P., Ramirez-Parra, E., Castellano, M. M., and Gutierrez, C. 2002. Interaction of geminivirus Rep protein with replication factor $\mathrm{C}$ and its potential role during geminivirus DNA replication. Virology 302:83-94.

Ma, Y., Zhou, T., Hong, Y., Fan, Z., and Li, H. 2008. Decreased level of ferredoxin I in Tobacco mosaic virus-infected tobacco is associated with development of the mosaic symptom. Physiol. Mol. Plant Pathol. 72: 39-45.

Martin, K., Singh, J., Hill, J. H., Whitham, S. A., and Cannon, S. B. 2016. Dynamic transcriptome profiling of Bean common mosaic virus (BCMV) infection in common bean (Phaseolus vulgaris L.). BMC Genomics 17:613.

Mazouni, K., Domain, F., Chauvat, F., and Cassier-Chauvat, C. 2003. Expression and regulation of the crucial plant-like ferredoxin of cyanobacteria. Mol. Microbiol. 49:1019-1029.

Mitra, S., and Baldwin, I. T. 2008. Independently silencing two photosynthetic proteins in Nicotiana attenuata has different effects on herbivore resistance. Plant Physiol. 148:1128-1138.

Mitra, S., and Baldwin, I. T. 2014. RuBPCase activase (RCA) mediates growth-defense trade-offs: Silencing RCA redirects jasmonic acid (JA) flux from JA-isoleucine to methyl jasmonate (MeJA) to attenuate induced defense responses in Nicotiana attenuata. New Phytol. 201 1385-1395.

Mubin, M., Amin, I., Amrao, L., Briddon, R. W., and Mansoor, S. 2010. The hypersensitive response induced by the V2 protein of a monopartite begomovirus is countered by the C2 protein. Mol. Plant Pathol. 11:245-254.

Nejat, N., Cahill, D. M., Vadamalai, G., Ziemann, M., Rookes, J., and Naderali, N. 2015. Transcriptomics-based analysis using RNA-Seq of the coconut (Cocos nucifera) leaf in response to yellow decline phytoplasma infection. Mol. Genet. Genomics 290:1899-1910.

Oka, K., Kobayashi, M., Mitsuhara, I., and Seo, S. 2013. Jasmonic acid negatively regulates resistance to Tobacco mosaic virus in tobacco. Plant Cell Physiol. 54:1999-2010.

Pandey, S. P., Roccaro, M., Schön, M., Logemann, E., and Somssich, I. E. 2010. Transcriptional reprogramming regulated by WRKY18 and WRKY40 facilitates powdery mildew infection of Arabidopsis. Plant J. 64:912-923.

Park, J. M., Park, C. J., Lee, S. B., Ham, B. K., Shin, R., and Paek, K. H. 2001. Overexpression of the tobacco Tsil gene encoding an EREBP/AP2-type transcription factor enhances resistance agains pathogen attack and osmotic stress in tobacco. Plant Cell 13:1035-1046.

Pontier, D., Godiard, L., Marco, Y., and Roby, D. 1994. hsr203J, a tobacco gene whose activation is rapid, highly localized and specific for incompatible plant/pathogen interactions. Plant J. 5:507-521.

Qian, Y., Hou, H., Shen, Q., Cai, X., Sunter, G., and Zhou, X. 2016. RepA protein encoded by Oat dwarf virus elicits a temperature-sensitive hypersensitive response-type cell death that involves jasmonic aciddependent signaling. Mol. Plant-Microbe Interact. 29:5-21.

Qin, X., Wu, H., Chen, J., Wu, L., Lin, S., Khan, M. U., Boorboori, M. R., and Lin, W. 2017. Transcriptome analysis of Pseudostellaria heterophylla in response to the infection of pathogenic Fusarium oxysporum. BMC Plant Biol. 17:155.

Rahoutei, J., Garcia-Luque, I., and Baron, M. 2000. Inhibition of photosynthesis by viral infection: Effect on PSII structure and function Physiol. Plant. 110:286-292.

Rodamilans, B., San León, D., Mühlberger, L., Candresse, T., Neumüller, M., Oliveros, J. C., and García, J. A. 2014. Transcriptomic analysis of Prunus domestica undergoing hypersensitive response to Plum pox virus infection. PLoS One 9:e100477.

Rodrigues, C. M., de Souza, A. A., Takita, M. A., Kishi, L. T., and Machado, M. A. 2013. RNA-Seq analysis of Citrus reticulata in the early stages of Xylella fastidiosa infection reveals auxin-related genes as a defense response. BMC Genomics 14:676.

Ryšlavá, H., Müller, K., Semorádová, S., Synková, H., and Čeřovská, N. 2003. Photosynthesis and activity of phosphoenolpyruvate carboxylase 
in Nicotiana tabacum L. leaves infected by Potato virus A and Potato virus $Y$. Photosynthetica 41:357-363.

Schubert, J., Habekuss, A., Kazmaier, K., and Jeske, H. 2007. Surveying cereal-infecting geminiviruses in Germany-Diagnostics and direct sequencing using rolling circle amplification. Virus Res. 127:61-70.

Serrano, I., Audran, C., and Rivas, S. 2016. Chloroplasts at work during plant innate immunity. J. Exp. Bot. 67:3845-3854.

Sharma, P., and Ikegami, M. 2010. Tomato leaf curl Java virus V2 protein is a determinant of virulence, hypersensitive response and suppression of posttranscriptional gene silencing. Virology 396:85-93.

Shen, Q., Liu, Z., Song, F., Xie, Q., Hanley-Bowdoin, L., and Zhou, X. 2011. Tomato SISnRK1 protein interacts with and phosphorylates $\beta C 1$, a pathogenesis protein encoded by a geminivirus $\beta$-satellite. Plant Physiol. 157:1394-1406.

Sood, A., and Chauhan, R. S. 2017. Comparative NGS Transcriptomics unravels molecular components associated with mosaic virus infection in a bioenergy plant species, Jatropha curcas L. BioEnergy Res. 10:129-145.

Su, Y., Hong, C., and Lin, Y. 2014. Plant ferredoxin-like protein enhances resistance to bacterial soft rot disease through PAMP-triggered immunity in Arabidopsis thaliana. Eur. J. Plant Pathol. 140:377-384.

Tang, K., Sun, X., Hu, Q., Wu, A., Lin, C., Lin, H., Twyman, R. M., Christou, P., and Feng, T. 2001. Transgenic rice plants expressing the ferredoxin-like protein (AP1) from sweet pepper show enhanced resistance to Xanthomonas oryzae pv. oryzae. Plant Sci. 160:1035-1042.

Thiel, H., Hleibieh, K., Gilmer, D., and Varrelmann, M. 2012. The P25 pathogenicity factor of Beet necrotic yellow vein virus targets the sugar beet $26 \mathrm{~S}$ proteasome involved in the induction of a hypersensitive resistance response via interaction with an F-box protein. Mol. PlantMicrobe Interact. 25:1058-1072.

Thordal-Christensen, H., Zhang, Z., Wei, Y., and Collinge, D. B. 1997. Subcellular localization of $\mathrm{H}_{2} \mathrm{O}_{2}$ in plants. $\mathrm{H}_{2} \mathrm{O}_{2}$ accumulation in papillae and hypersensitive response during the barley-powdery mildew interaction. Plant J. 11:1187-1194.

Ueda, H., Yamaguchi, Y., and Sano, H. 2006. Direct interaction between the Tobacco mosaic virus helicase domain and the ATP-bound resistance protein, $\mathrm{N}$ factor during the hypersensitive response in tobacco plants. Plant Mol. Biol. 61:31-45.

Vorst, O., van Dam, F., Weisbeek, P., and Smeekens, S. 1993. Lightregulated expression of the Arabidopsis thaliana ferredoxin A gene involves both transcriptional and post-transcriptional processes. Plant J. 3:793-803.

Wang, Y., Yu, B., Zhao, J., Guo, J., Li, Y., Han, S., Huang, L., Du, Y., Hong, Y., Tang, D., and Liu, Y. 2013. Autophagy contributes to leaf starch degradation. Plant Cell 25:1383-1399.

Wang, Z., Gerstein, M., and Snyder, M. 2009. RNA-Seq: A revolutionary tool for transcriptomics. Nat. Rev. Genet. 10:57-63.

Xie, Q., Suárez-López, P., and Gutiérrez, C. 1995. Identification and analysis of a retinoblastoma binding motif in the replication protein of a plant DNA virus: Requirement for efficient viral DNA replication. EMBO J. 14:4073-4082.

Zhao, J., Zhang, X., Hong, Y., and Liu, Y. 2016. Chloroplast in plant-virus interaction. Front. Microbiol. 7:1565.

Zou, J., Rodriguez-Zas, S., Aldea, M., Li, M., Zhu, J., Gonzalez, D. O., Vodkin, L. O., DeLucia, E., and Clough, S. J. 2005. Expression profiling soybean response to Pseudomonas syringae reveals new defense-related genes and rapid HR-specific downregulation of photosynthesis. Mol. Plant-Microbe Interact. 18:1161-1174.

Zurbriggen, M. D., Carrillo, N., Tognetti, V. B., Melzer, M., Peisker, M., Hause, B., and Hajirezaei, M. R. 2009. Chloroplast-generated reactive oxygen species play a major role in localized cell death during the nonhost interaction between tobacco and Xanthomonas campestris pv. vesicatoria. Plant J. 60:962-973.

\section{AUTHOR-RECOMMENDED INTERNET RESOURCES}

Transcriptome reference database: http://solgenomics.net/ BLASTX algorithm: https://www.ncbi.nlm.nih.gov/ Blast2go software: http://www.blast2go.com/ Inter-ProScan: http://www.ebi.ac.uk/Tools/pfa/iprscan/ 